\title{
High-resolution imaging and near-infrared spectroscopy of penumbral decay
}

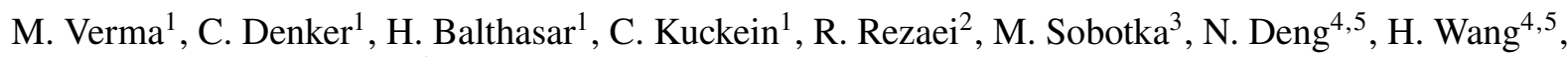 \\ A. Tritschler ${ }^{6}$, M. Collados ${ }^{2}$, A. Diercke ${ }^{1,7}$, and S. J. González Manrique ${ }^{1,8}$ \\ ${ }^{1}$ Leibniz-Institut für Astrophysik Potsdam (AIP), An der Sternwarte 16, 14482 Potsdam, Germany \\ e-mail: mverma@aip.de \\ 2 Instituto de Astrofísica de Canarias, C/ Vía Láctea s/n, 38205 La Laguna, Tenerife, Spain \\ 3 Astronomical Institute, Academy of Sciences of the Czech Republic, Fričova 298, 25165 Ondřejov, Czech Republic \\ ${ }^{4}$ Space Weather Research Laboratory, New Jersey Institute of Technology, University Heights Newark, New Jersey, USA \\ ${ }^{5}$ Big Bear Solar Observatory, Big Bear City, California, USA \\ ${ }^{6}$ National Solar Observatory, 3665 Discovery Drive, Boulder, CO 80303, USA \\ ${ }^{7}$ Universität Potsdam, Institut für Physik und Astronomie, Karl-Liebknecht-Straße 24/25, 14476 Potsdam-Golm, Germany \\ ${ }^{8}$ Astronomical Institute of the Slovak Academy of Sciences, 05960 Tatranská Lomnica, Slovakia
}

Received 19 August 2017 / Accepted 9 January 2018

\begin{abstract}
Aims. Combining high-resolution spectropolarimetric and imaging data is key to understanding the decay process of sunspots as it allows us to scrutinize the velocity and magnetic fields of sunspots and their surroundings.

Methods. Active region NOAA 12597 was observed on 2016 September 24 with the 1.5-meter GREGOR solar telescope using high-spatial-resolution imaging as well as imaging spectroscopy and near-infrared (NIR) spectropolarimetry. Horizontal proper motions were estimated with local correlation tracking, whereas line-of-sight (LOS) velocities were computed with spectral line fitting methods. The magnetic field properties were inferred with the "Stokes Inversions based on Response functions" (SIR) code for the Si I and Ca I NIR lines.

Results. At the time of the GREGOR observations, the leading sunspot had two light bridges indicating the onset of its decay. One of the light bridges disappeared, and an elongated, dark umbral core at its edge appeared in a decaying penumbral sector facing the newly emerging flux. The flow and magnetic field properties of this penumbral sector exhibited weak Evershed flow, moat flow, and horizontal magnetic field. The penumbral gap adjacent to the elongated umbral core and the penumbra in that penumbral sector displayed LOS velocities similar to granulation. The separating polarities of a new flux system interacted with the leading and central part of the already established active region. As a consequence, the leading spot rotated $55^{\circ}$ clockwise over $12 \mathrm{~h}$.

Conclusions. In the high-resolution observations of a decaying sunspot, the penumbral filaments facing the flux emergence site contained a darkened area resembling an umbral core filled with umbral dots. This umbral core had velocity and magnetic field properties similar to the sunspot umbra. This implies that the horizontal magnetic fields in the decaying penumbra became vertical as observed in flare-induced rapid penumbral decay, but on a very different time-scale.
\end{abstract}

Key words. Sun: photosphere - sunspots - Sun: magnetic fields - Sun: infrared - techniques: imaging spectroscopy techniques: spectroscopic

\section{Introduction}

The formation of sunspots is a relatively rapid process (e.g., Leka \& Skumanich 1998) compared to their lifetime. Theory suggests that a pore develops a penumbra when the magnetic field reaches an inclination of $45^{\circ}$ at its edges (Rucklidge et al. 1995; Tildesley \& Weiss 2004). On the other hand, penumbral decay is a slow process, unless it is related to solar flares or other eruptive phenomena; for example, rapid penumbral decay was observed following X-class solar flares (Wang et al. 2004; Deng et al. 2005).

The flow and magnetic fields of decaying sunspots, despite being the subject of many observational and theoretical studies, still lack a comprehensive physical description. Various aspects of sunspot decay were extensively studied (e.g., Meyer et al. 1974; McIntosh 1981; Martínez Pillet 2002; Solanki 2003). Stable leading sunspots and irregular trailing sunspots have different decay rates (Martínez Pillet 2002). Various decay laws have been put forward to explain the decay rates, such as a linear decay law by Bumba (1963), and a parabolic decay by Petrovay \& van Driel-Gesztelyi (1997). These decay laws were reviewed by Martínez Pillet (2002), including diffusion models to explain how magnetic flux is distributed over a larger area when a spot decays. However, according to the latter author, the flux removal process still needs a satisfactory explanation.

Rempel (2015) numerically simulated a sunspot (with penumbra) and a naked sunspot (penumbra removed after $20 \mathrm{~h}$ ) to analyze how the presence of a penumbra influences the moat flow and sunspot decay. He found large-scale flows surrounding both spots with the exception of the Evershed flow, which should only exist in the presence of a penumbra. In these simulations, the submergence of the horizontal magnetic field was the dominant decay process. A strong reduction of the downflow filling factor and convective rms-velocity, underneath the sunspot penumbra and the outer boundary of the naked spot, were the two factors in the simulation that inhibited the decay process. 
Bellot Rubio et al. (2008) discovered finger-like structures near a sunspot in the latter stages of evolution. These features were small-scale inhomogeneities in the magnetic canopy and were neither related to the penumbral filament nor to the Evershed flow. The authors speculated that these structures are related to penumbral field lines that no longer carry strong Evershed flows and rise to the chromosphere, thus producing the disappearance of the penumbra at photospheric levels. Moving magnetic features (MMFs) are present around many decaying sunspots (e.g., Harvey \& Harvey 1973). Verma et al. (2012) noted that flux carried by MMFs from the decaying spot reaches the surrounding supergranular boundary, similar to what is described by Deng et al. (2007). However, the relation between penumbral filaments, MMFs, Evershed flow, and moat flow is still a matter of debate (e.g., Sainz Dalda \& Martínez Pillet 2005; Cabrera Solana et al. 2006).

The changes in the size and the magnetic/velocity field of umbra and penumbra can give some indication, when sunspots begin to form or decay. Around a decaying sunspot, Balthasar et al. (2013) reported a reversed gradient in the vertical magnetic field component in the penumbra. The authors interpret it as an inclination affect, which is already seen in Balthasar \& Gömöry (2008). This height dependence was derived by comparing the magnetic field obtained from the infrared lines Fe I $1078.3 \mathrm{~nm}$ and Si I $1078.6 \mathrm{~nm}$. Watanabe et al. (2014) observed formation and decay of a rudimentary penumbra in a protospot (Leka \& Skumanich 1998), where the penumbra developed at the expense of umbral magnetic flux. While the penumbra decayed, they observed that the penumbral field became vertical resulting in the recovery of umbral area, that is, penumbral decay leads to rearrangement of magnetic field lines. In contrast, Schlichenmaier et al. (2010) noticed that the umbral area remained constant when the sunspot was developing a penumbra. Based on a study of ten sunspots, Jurčák (2011) concluded that the inner penumbral boundaries are defined by the critical value of the vertical component of the magnetic field, that is, $B_{\text {stable }}=1.8 \mathrm{kG}$. Recently, Jurčák et al. (2017) studied penumbra formation around a pore, which supports the scenario proposed earlier by Jurčák (2011) and Jurčák et al. (2015), that is, the stable vertical component of magnetic field is needed to establish the umbra-penumbra boundary.

The magnetic field in the vicinity of sunspots also plays a significant role in formation and decay of sunspot penumbra. Künzel (1969) found that the polarity, magnetic field strength, and size of a neighboring spot influence the penumbra of the sunspot. He also noticed that no penumbra forms between spots with identical polarity. Flux emergence in the vicinity of a sunspot inhibits the formation of a stable penumbra, as demonstrated by Schlichenmaier et al. (2010) and Rezaei et al. (2012). Both studies agree that stable penumbral filaments only form away from the flux emergence site, implying a "quiet" environment as a prerequisite for developing a penumbra. Lim et al. (2013) observed the formation of a non-radial penumbra in a flux-emergence region with pre-existing chromospheric canopy fields. They suggested that in an emerging region, the penumbra is formed when the emerging flux is constrained from developing any further by the overlying chromospheric canopy fields. However, Murabito et al. (2017) observed that a stable penumbra was formed in a sunspot at the site facing the opposite polarity and the region of flux emergence. These sometimes contradictory findings lead to the question: what role does magnetic flux emergence and its interaction with the overlying canopy play in the decay of sunspot penumbra? The present work contributes to answering this open question. We present high-resolution GREGOR observations of a decaying sunspot, where the decaying penumbra faces the flux emergence site.

\section{Observations and data reduction}

\subsection{Observations}

The aim of the 2016 September coordinated observing campaign was to obtain high-resolution spectropolarimetric data and accurate measurements of the photospheric and chromospheric three-dimensional magnetic and flow fields within an active region. The campaign made use of the following telescopes and instruments: the GREGOR Fabry-Pérot Interferometer (GFPI; Puschmann et al. 2012; Denker et al. 2010, and references therein), the High-resolution Fast Imager (HiFI; Kuckein et al. 2017), and the GREGOR Infrared Spectrograph (GRIS; Collados et al. 2012) at the 1.5-meter GREGOR solar telescope (Schmidt et al. 2012; Denker et al. 2012); the Echelle Spectrograph at the Vacuum Tower Telescope (VTT; von der Lühe 1998); the Spectro-Polarimeter (SP; Ichimoto et al. 2008) of the Solar Optical Telescope (SOT; Tsuneta et al. 2008) on board the Japanese space mission Hinode (Kosugi et al. 2007); and the Helioseismic and Magnetic Imager (HMI; Scherrer et al. 2012; Schou et al. 2012) and the Atmospheric Imaging Assembly (AIA; Lemen et al. 2012) on board the Solar Dynamics Observatory (SDO; Pesnell et al. 2012). The observations were carried out for 10 days. This study is based on just one of the datasets containing a decaying sunspot, which was observed with GFPI, HiFI, and GRIS. Continuum images and magnetograms of HMI provide context information.

Active region NOAA 12597 emerged on the solar surface on 2016 September 22. It was classified as a simple bipolar $\beta$-region with a strong leading spot and scattered pores forming the trailing part. By 00:00 UT on 2016 September 24, the leading sunspot was encircled by a complete penumbra. The trailing part contained two large pores. The GREGOR observations started two days later on 2016 September 24. The region already crossed the central meridian and was located at coordinates $\left(110^{\prime \prime},-350^{\prime \prime}\right)$ corresponding to $\mu \approx 0.92$ on the solar disk. During the observations the mature leading sunspot started to decay. The region was relatively quiet and only two B-class solar flares occurred, one before and one after the GREGOR observations.

The leading sunspot of the region was observed with the GFPI in spectroscopic mode starting at 08:52 UT on 2016 September 24. The instrument scanned the photospheric Fe I $\lambda 617.3 \mathrm{~nm}$ line. The imaging spectrometer recorded 140 scans in total with 25 equidistantly spaced wavelength points. One spectral scan took about $25 \mathrm{~s}$ with an exposure time of $10 \mathrm{~ms}$ and eight frames at each wavelength point. In addition, strictly simultaneous broad-band images were captured using an interference filter with a full-width-at-half-maximum (FWHM) of $10 \mathrm{~nm}$ centered at $\lambda 612.3 \mathrm{~nm}$. Broad- and narrow-band images have $688 \times 512$ pixels after $2 \times 2$-pixel binning with an effective image scale of about $0.081^{\prime \prime}$ pixel $^{-1}$ resulting in a field-of-view (FOV) of about $56^{\prime \prime} \times 41^{\prime \prime}$.

The two HiFI cameras recorded strictly simultaneous highresolution $G$-band $\lambda 430.7 \mathrm{~nm}$ and blue continuum $\lambda 450.5 \mathrm{~nm}$ images with an exposure time of $1.8 \mathrm{~ms}$. Initially, sets of 500 frames with a frame rate of $47 \mathrm{~Hz}$ were acquired but ultimately, after frame selection, only the best 100 calibrated frames were stored for post-processing and image restoration. The sCMOS imagers have a pixel size of $6.5 \mu \mathrm{m} \times 6.5 \mu \mathrm{m}$. The 
images contain $2560 \times 2160$ pixels, and the image scale is about $0.025^{\prime \prime}$ pixel $^{-1}$, which leads to a FOV of about $65^{\prime \prime} \times 55^{\prime \prime}$. The total duration of the HiFI time-series was about $46 \mathrm{~min}$. Only HiFI blue continuum images are used in this study to provide high-resolution context information.

In parallel to GFPI and HiFI, we observed in the $\mathrm{He}$ I $\lambda 1083.0 \mathrm{~nm}$ spectral region with GRIS recording spectropolarimetric data. This NIR spectral window contains various solar and telluric lines. However, of primary interest are the photospheric Si I $\lambda 1082.7 \mathrm{~nm}$ line, the chromospheric He I $\lambda 1083.0 \mathrm{~nm}$ triplet (two blended lines $\lambda 1083.030 \mathrm{~nm}$ and $\lambda 1083.025 \mathrm{~nm}$ ), and the photospheric Ca I $\lambda 1083.9 \mathrm{~nm}$ line. The slit-width was about $0.25^{\prime \prime}$. The number of spectral points was $n=1010$, and the spectral sampling was $1.81 \mathrm{pm}^{\text {pixel }}{ }^{-1}$. The scan covered $51.8^{\prime \prime}$ in 360 steps with a step size of about $0.144^{\prime \prime}$. The image scale along the slit was about $0.136^{\prime \prime}$ pixel $^{-1}$ resulting in a FOV of about $62^{\prime \prime} \times 52^{\prime \prime}$. The integration time used for each slit position was $100 \mathrm{~ms}$ with ten accumulations. Thus, the first scan of the sunspot with 360 steps took $36 \mathrm{~min}$, and the second with 300 steps finished in $30 \mathrm{~min}$. Only the first scan starting at 09:02 UT is analyzed in detail, while the second scan starting at 10:30 UT provides supporting context information. Since the installation of a three-mirror system as de-rorator in the GREGOR, the observed images and spectra no longer have to be de-rotated and can easily be matched with orientation of the SDO images.

\subsection{Data reduction and analysis}

Calibration of GFPI and HiFI data was carried out with the data processing pipeline "sTools" (Kuckein et al. 2017). The sTools pipeline performs basic data calibration such as dark-frame subtraction and flat-field corrections for both post-focus instruments. In addition, for GFPI data it computes and applies prefilter transmission and blueshift corrections along with removing the tilt in the spectral profile by matching it with the Fourier Transform Spectrometer (FTS; Neckel \& Labs 1984) spectral atlas. After basic calibration, sTools also performs the image restorations providing us with Level 2 data. Narrow- and broadband images were restored using Multi-Object Multi-Frame Blind Deconvolution (MOMFBD; Löfdahl 2002; van Noort et al. 2005). The Kiepenheuer Institute speckle interferometry package (KISIP; Wöger \& von der Lühe 2008; Wöger et al. 2008) was used to restore the blue continuum and $G$-band images. For both GFPI and HiFI images, the image contrast, degraded by scattered light, was then corrected using the method described in Bello González \& Kneer (2008). One example of a restored blue continuum image is shown in Fig. 1. The restored HiFI images are finally written as image extensions in the Flexible Image Transport System (Wells et al. 1981; Hanisch et al. 2001) format for further use and analysis. The analysis and management for GREGOR data, specifically for image restoration and imaging spectroscopy, is described in detail in Denker et al. (2018).

The GFPI spectra of the Fe I $\lambda 617.3 \mathrm{~nm}$ line provided us with many spectral line characteristics including FWHM, equivalent width, and line-shift or LOS velocity. The most important quantity is the LOS velocity in and around the sunspot. We determined the velocity with a Fourier phase method (Schmidt et al. 1999). We chose this method as it utilizes the entire line profile, takes into consideration the spectral line asymmetry, and is less sensitive to noise. The line shifts calculated with this method were then converted into velocities using the Doppler formula. We used the average photospheric velocity of quietSun granulation as the frame of reference. In the computed

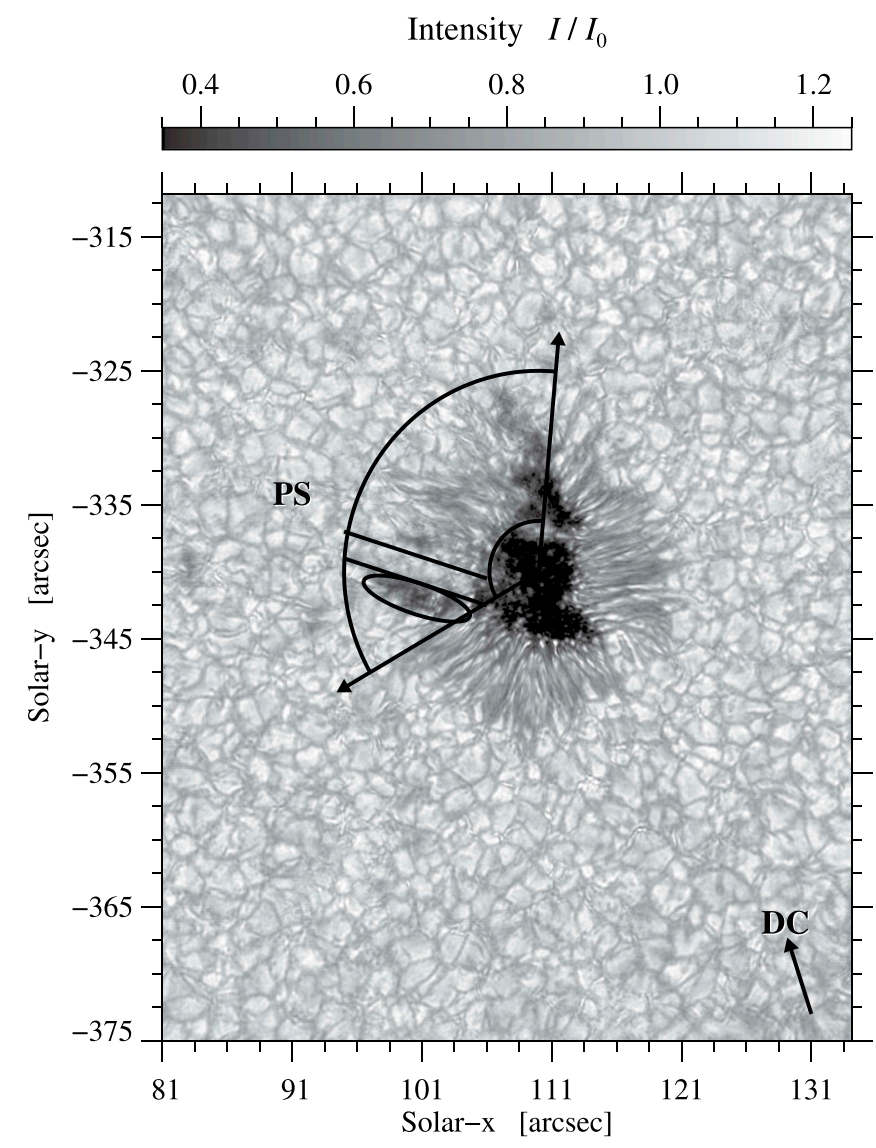

Fig. 1. Speckle-restored blue continuum image from HiFI observed at 08:50 UT on 2016 September 24. The two black arcs mark the penumbral sector PS discussed in Sect. 3. The arrow in the lower right corner points to solar-disk center DC. The black oval and the two parallel lines inside the PS indicate the elongated, dark umbral core and the penumbral gap, respectively, which are discussed in Sect. 3.

LOS velocity map redshifts are positive and blueshifts are negative.

The synoptic nature and good time coverage of SDO data complements the high-resolution observations and provides context information. The HMI continuum image and the LOS magnetogram, along with AIA EUV images in Fe IX $\lambda 17.1 \mathrm{~nm}$ and FUV images at $\lambda 160 \mathrm{~nm}$ present an overview of the observed active region (Fig. 2). The FOVs covered by various instruments are indicated by colored rectangular boxes. In addition, we used continuum images and LOS magnetograms for every hour on September 24 to study the temporal evolution of the region.

One of the advantages of having time-series of highresolution images at the same time as spectroscopic data is that we can determine horizontal proper motions as well as LOS velocities. The horizontal proper motions were computed from the restored GFPI broad-band images, where we applied our implementation (Verma \& Denker 2011; Verma et al. 2013) of the local correlation tracking (LCT; November \& Simon 1988) algorithm. The images in both time-series were aligned with sub-pixel accuracy with respect to average image of the timeseries and the signature of five-minute oscillations was removed using a subsonic Fourier filter with the photospheric sound speed as cut-off velocity. The LCT velocities were computed over the image tiles of $48 \times 48$ pixels with a Gaussian kernel with a $F W H M=1200 \mathrm{~km}$. Consecutive images were used to calculate LCT maps, that is, the cadence was $\Delta t=25 \mathrm{~s}$, and 


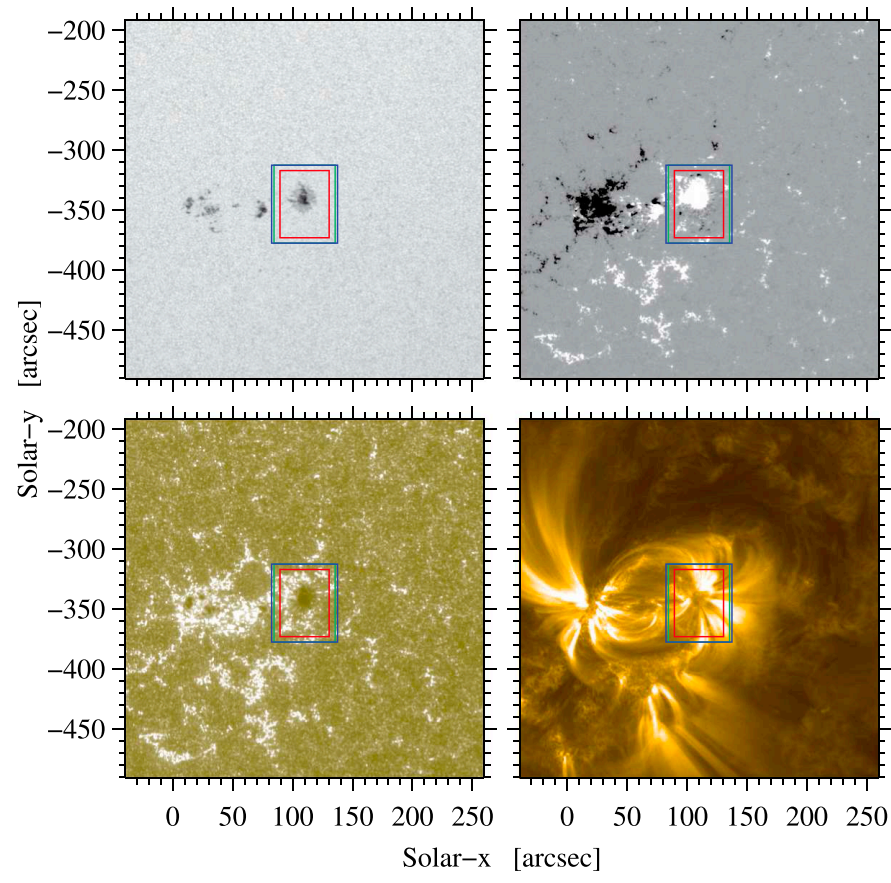

Fig. 2. Overview of active region NOAA 12597: HMI continuum image (top-left), HMI magnetogram (top-right), AIA $\lambda 160 \mathrm{~nm}$ image (bottom-left), and AIA Fe IX $\lambda 17.1 \mathrm{~nm}$ image (bottom-right) observed at 09:00 UT on 2016 September 24. The red, green, and blue boxes represent the FOV of GFPI, GRIS scan, and HiFI images, respectively. The magnetogram was clipped between $\pm 250 \mathrm{G}$ and the AIA $\lambda 17.1 \mathrm{~nm}$ image is displayed on a linear scale.

the computed maps were then averaged over a time period of $\Delta T=40 \mathrm{~min}$.

The basic data reduction for GRIS is carried out on site using the GRIS data pipeline (Collados, priv., communication). The various steps include dark-frame subtraction, flat-field correction, crosstalk removal, and calibration of the polarization modulator (Collados 1999). Details of the next data reduction steps such as wavelength calibration and correction for the spectrograph profile, and so on are given in Verma et al. (2016). We used the "Stokes Inversion based on Response functions" (SIR, Ruiz Cobo \& del Toro Iniesta 1992) code to invert the GRIS spectra of the two scans. We restricted ourselves to the photospheric lines Si I $\lambda 1082.7 \mathrm{~nm}\left(g_{\mathrm{eff}}=1.5\right)$ and Ca I $\lambda 1083.9 \mathrm{~nm}$ $\left(g_{\text {eff }}=1.5\right)$. The inversion for two lines were performed separately because velocity and magnetic field were implemented independently of height. The starting model for Si I covered the optical depth range $+1.0 \leq \log \tau \leq-5.4$, whereas for Ca I the optical depth range was $+1.0 \leq \log \tau \leq-4.4$. For both scans a limb-darkening factor was considered according to Eq. (10) of Pierce \& Slaughter (1977). We assumed a constant macroturbulence of $1 \mathrm{~km} \mathrm{~s}^{-1}$ and $2 \mathrm{~km} \mathrm{~s}^{-1}$ for the Si I and Ca I lines, respectively. In addition, for both lines a fixed stray light contribution of two percent was used. This value was chosen as a lower limit (Balthasar et al. 2016). The inversions for both lines delivered the temperature stratification with three nodes $T(\tau)$, the total magnetic flux density $B_{\text {tot }}$, the magnetic inclination $\gamma$ and azimuth $\phi$, and the Doppler velocity $v_{\mathrm{LOS}}$ that is constant with height (one node for each of these physical parameters). The magnetic azimuth ambiguity for the inversions was solved by assuming the center of leading spot as the azimuth center (Balthasar 2006). Finally, we rotated the magnetic vector with respect to the local solar frame. Pixels for which the circular and the linear polarization were below $0.2 \%$ degree of polarization, were excluded from any further analysis of the magnetic field, as this value corresponds to the noise level of the polarization signal.

In addition to the SIR results, the LOS velocities and linecore intensities for the above mentioned Si I, Ca I, and He I lines were derived using a single Lorentzian model to fit the line core (González Manrique et al. 2016). The model is given by $L=a_{0} /\left(u^{2}+1\right)+a_{3}$ with $u=\left(x-a_{1}\right) / a_{2}$, where $a_{0}$ is the amplitude, $a_{1}$ is the peak centroid, $2 a_{2}$ is the FWHM, and $a_{3}$ is a constant representing the continuum. All the shown GRIS maps are trimmed and aligned to match the GFPI's FOV.

When computing the LOS velocities for all spectral lines, we considered quiet-Sun granulation as the frame of reference. The reference for the chromospheric He I line, was a quiet-Sun region, where additionally filamentary structures were absent. Because the photospheric lines exhibit convective blueshifts, we computed the velocity offsets based on the SIR results with respect to the umbra, which can be assumed at rest. The approximate velocity offsets are Fe I $-215 \mathrm{~m} \mathrm{~s}^{-1}, \mathrm{Ca} \mathrm{I}-300 \mathrm{~m} \mathrm{~s}^{-1}$, and Si I $-50 \mathrm{~m} \mathrm{~s}^{-1}$, which slightly depend on the selected part of the umbra. Here, we chose the entire umbra encircled by the black contours. However, other choices, for example the darkest part of the umbra, affected the offsets by only about $50 \mathrm{~m} \mathrm{~s}^{-1}$. As a result, the umbra appears redshifted in all photospheric LOS velocity maps but the fine structure of penumbra and granulation is easier to compare across different spectral line in this way. Our results agree with the trend for the convective blueshift as a function of line depth as given by, for example, Reiners et al. (2016).

\section{Results}

\subsection{Temporal evolution of the active region}

Studying the evolution of active region NOAA 12597 on 2016 September 24 is based on HMI continuum images and magnetograms along with HiFI images. On the day of the GREGOR observations, the sunspot group was a simple bipolar region with a weak negative-polarity trailing part and a positive-polarity leading sunspot. The observations focused on the leading mature sunspot. Figures 3 and 4 contain $150^{\prime \prime} \times 80^{\prime \prime}$-snapshots of the region, where the continuum images and LOS magnetograms are displayed at two-hour intervals starting from 01:00 UT on 2016 September 24. The whole region can be divided into two flux systems: a large decaying active region (DAR) and a small emerging active region (EAR) to the south of the DAR's trailing part. The relevant spots and pores are labeled in the 09:00 UT panel of Figs. 3 and 4 to clearly identify them for the subsequent analysis and discussion, that is, the main leading spot $P_{1}$, the trailing pores $N_{1}$ and $N_{2}$, the central negative pore $N_{3}$, the leading pore $p_{1}$, and the trailing pore $n_{1}$ in the EAR. The nomenclature incorporates both magnetic polarity and membership with respect to DAR and EAR. The membership to DAR and EAR was assigned based on the evolution of the region seen in the time-lapse movies of SDO. Since the indication of $N_{3}$ was already there in the magnetogram at 01:00 UT before the EAR started to develop, we ascribed it to the DAR.

At 01:00 UT, $\mathrm{P}_{1}$ displayed two light bridges indicating the onset of its fragmentation. In the next two hours at 03:00 UT, $P_{1}$ remained unchanged. At 05:00 UT, the penumbral sector PS (marked in Fig. 2) of $P_{1}$, which faced the trailing region, started to decay. A gap appeared at the edge of lower light 
M. Verma et al.: High-resolution imaging and near-infrared spectroscopy of penumbral decay
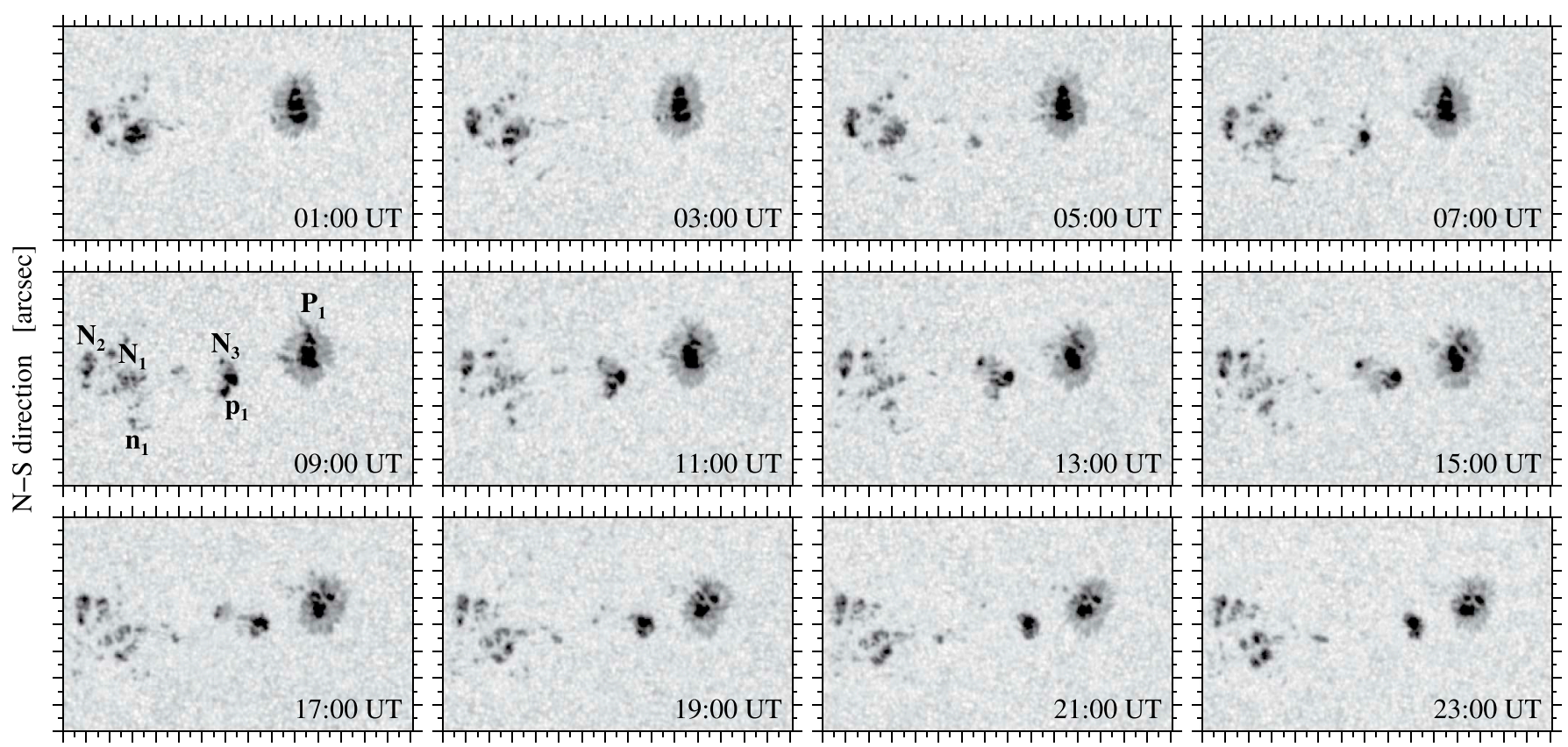

E-W direction [arcsec]

Fig. 3. Evolution of active region NOAA 12597 on 2016 September 24 based on HMI continuum images with a FOV of $150^{\prime \prime} \times 80^{\prime \prime}$. Major tick marks are placed at 10"-intervals. The images were displayed at two-hour intervals starting at 01:00 UT. The abbreviations in the 09:00 UT panel indicate the leading spot $P_{1}$, the trailing pores $N_{1}$ and $N_{2}$, the central negative pore $N_{3}$, and the leading and trailing pores $p_{1}$ and $n_{1}$ of the emerging active region.
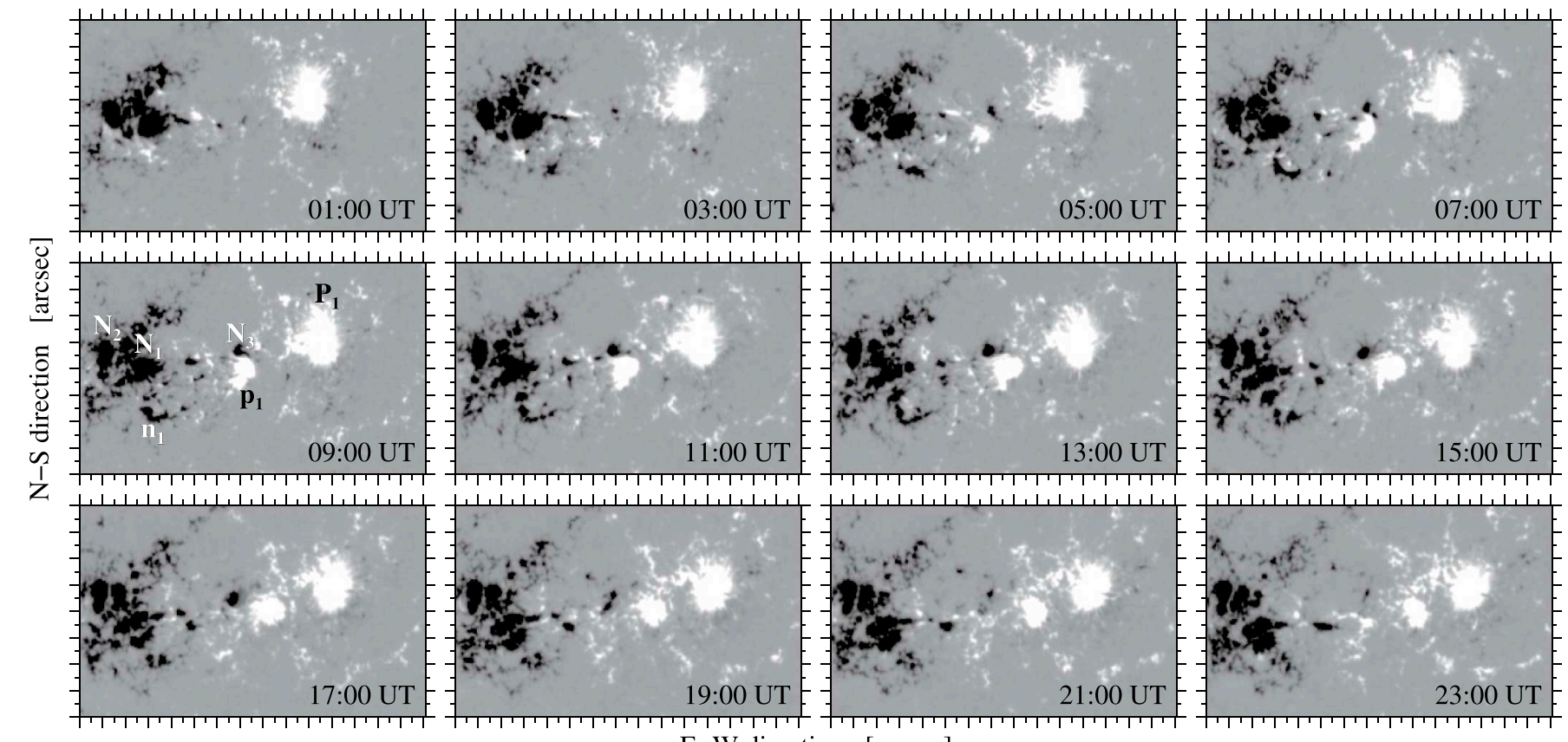

E-W direction [arcsec]

Fig. 4. Evolution of active region NOAA 12597 on 2016 September 24 based on HMI magnetograms, which were scaled between \pm 500 G. Annotations, labels, and FOV are the same as in Fig. 3.

bridge of $P_{1}$. At 07:00 UT, this gap resembled umbral cores and stretched out towards the trailing polarity in the magnetogram. In addition, the lower light bridge in $P_{1}$ disappeared. At 09:00 UT, the GREGOR observations started. The spot $P_{1}$ now contained two elongated features like umbral cores within PS - one on top and the other on the side facing the trailing part. Only the upper light bridge remained. The high-resolution HiFI images revealed further details within $P_{1}$ (Fig. 2). Both
$G$-band and blue-continuum images exhibited umbral dots at the location of the disappearing light bridge. The dark extrusion, which resembled an umbral core, was located at the edge of this light bridge (black oval in Fig. 2). In the gap, between the umbral core and the penumbral sector (indicated in Fig. 2 by two parallel lines), granulation was present. The upper light bridge started to fork creating a $\mathrm{Y}$-shape. The $G$-band images reveal many $G$-band bright points in the surroundings indicative 
of small-scale flux elements. The time-series of GFPI broadband images uncover many minutes and fast changes in the spot, which are discussed in Sect. 3.2. At 11:00 UT, $P_{1}$ exhibited clear signs of penumbral decay. Comparing earlier images with those at 13:00 UT, $P_{1}$ distinctly rotated, which is evident when examining position and direction of the light bridge. In the four hours from 09:00-13:00 UT, the sunspot rotated $30^{\circ}$ clockwise with a rotation rate of about $15^{\circ}$ every two hours. At this time, PS almost decayed. This process continued for the next two hours as seen in the continuum images and magnetograms at 15:00 UT. The rotation of $P_{1}$ continued and became more prominent. By 17:00 UT, the rotation of $P_{1}$ was very obvious, and new light bridges appeared. However, the rotation rate had slowed down. In the last four hours of the day, $\mathrm{P}_{1}$ became more compact, that is, with a decreasing penumbral coverage, with stronger light bridges, and with substantial rotation of almost $55^{\circ}$ in clockwise direction with respect to the image at 09:00 UT. In the beginning, at 01:00 UT, the trailing part consisted of two big pores with $\mathrm{N}_{1}$ on the south having a rudimentary penumbra and $\mathrm{N}_{2}$ on the north, along with some scattered pores. The region between the two main polarities contained only granulation. However, in the corresponding magnetogram, small-scale mixed polarities were present at that location. In next two hours at 03:00 UT, $\mathrm{N}_{1}$ developed a light bridge. Over the next hours, continuum images revealed that the trailing pores started to disintegrate. By the end of the day, the trailing part of the active region shrunk with few small scattered pores remaining.

The central part of the active region contained only granulation at 01:00 UT. Some small-scale mixed-polarity patches near the trailing pores were still too weak to leave an imprint in the continuum images. The central negative pore $N_{3}$ belonging to DAR was not visible in the continuum images until 05:00 UT. However, its presence was already noticeable in the magnetograms at 03:00 UT. Throughout the day the central pore mainly interacted with the positive polarity of the EAR, which appeared as separate magnetic flux-bundle rising onto the surface at the lower right-side of $N_{1}$. Signs of flux emergence were present in the 03:00 UT magnetograms, where the infant stages of two polarities were first evident. The leading and trailing part of the EAR developed at 05:00 UT as positive $p_{1}$ and negative $n_{1}$ polarity pores, respectively. At 07:00 UT, the center of the EAR contained many mixed polarity features. In addition, as often seen in flux-emergence regions, $p_{1}$ separated and moved away from $n_{1}$. In this process, $p_{1}$ moved closer to $N_{3}$. By 09:00 UT, $p_{1}$ grew in size by coalescence of small-scale magnetic features while $\mathrm{N}_{3}$ remained in close proximity. At 11:00 UT, both magnetograms and continuum images showed that $p_{1}$ and $N_{3}$ slid past each other like two cars passing each other along the central divider on a highway. This motion had an immediate affect on $P_{1}$ and resulted in the clockwise rotation of the whole leading sunspot.

In addition, the opposite-polarity pores, $p_{1}$ and $n_{1}$, were still separating and moving away from each other. At 13:00 UT, the most dynamic location was still the center of the FOV. There, $\mathrm{N}_{3}$ moved towards the trailing part and $\mathrm{p}_{1}$ towards the leading part of the DAR, which continued at 13:00 UT. The declining phase of the EAR began at 17:00 UT. The central pores $p_{1}$ and $\mathrm{N}_{3}$ were clearly separated. The trailing part of the EAR already decayed. Within the next two hours, $p_{1}$ and $N_{3}$ moved closer to the leading and trailing parts, respectively. Although, now only faint signatures of $\mathrm{N}_{3}$ were visible in continuum images. Mixed polarities were still present in the region of flux emergence, but the overall photometric morphology and magnetic topology significantly simplified.

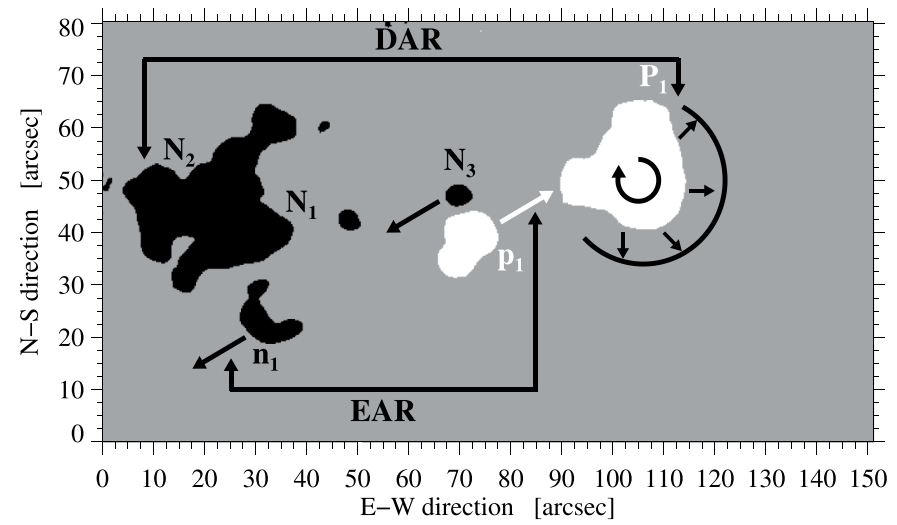

Fig. 5. Sketch based on the HMI magnetogram observed at 09:00 UT on 2016 September 24. Black and white areas mark the negative and positive polarities, respectively. Arrows indicate the direction in which different features moved over the course of the day. The black semicircle denotes the region around $P_{1}$ with moat flow and MMFs. The black lines with vertical arrows mark the extent and features belonging to DAR and EAR, also labeled with upper- and lowercase letters.

We used AIA $\lambda 171 \mathrm{~nm}$ EUV images to examine the topology of the coronal loop structure related to the active region. Early on 2016 September 24, the loops had a bipolar active region structure, that is, with arches connecting the two opposite polarities of the DAR, which were slightly lopsided towards the leading sunspot $P_{1}$. Onwards 04:00 UT, with the emergence of new flux, a low-lying loop structure connecting $p_{1}$ and $n_{1}$ was established at its location, disrupting the DAR loop structure. The flux emergence caused some small-scale brightenings and a small disruption in the large overarching loops. However, after the initial flux emergence phase the usual large bipolar arches reestablished themselves. The rotation of $P_{1}$ in photospheric layers caused the rotation of this foot point. However, the trailing polarity footpoint remained stable. In addition, loops terminating at $P_{1}$ expanded, slightly rotated, fanned out, and detached towards the western limb.

We summarized the main dynamical properties of the region in the sketch depicted in Fig. 5. The sketch is based on the magnetogram at 09:00 UT, which was smoothed and clipped at $\pm 250 \mathrm{G}$. All features are labeled as in Figs. 3 and 4. The main dynamics within the active region were indicated by arrows, that is, the separation of $p_{1}$ and $n_{1}$, the sliding of $N_{3}$ and $p_{1}$, and the resulting clockwise rotation of $P_{1}$. The arrows only show the direction but not the magnitude of these motions.

To quantify the overall decay rate of the leading spot, we followed the active region for five days and found that the region had recurring flux emergence at the same location. There were continuously merging flux elements in the trailing part. However, only the leading spot of the DAR, that is, $\mathrm{P}_{1}$ remained separated and did not merge with the neighboring emerging flux system. Hence, we computed flux and area growth and decay rates for only $\mathrm{P}_{1}$ covering five days, that is, September 22-27. We created a binary template based on HMI LOS magnetograms that contained only $\mathrm{P}_{1}$. We used a flux threshold of $500 \mathrm{G}$ along with morphological erosion using a 1-Mm kernel, followed by dilation using a 5-Mm kernel. To determine the photometric area, we used contrast-enhanced HMI continuum images. The sunspot was identified with the help of intensity thresholding, that is, $I_{\text {spot }}<0.95 I_{0}$, where $I_{0}$ refers to the normalized quiet-Sun intensity. The measured area and magnetic 


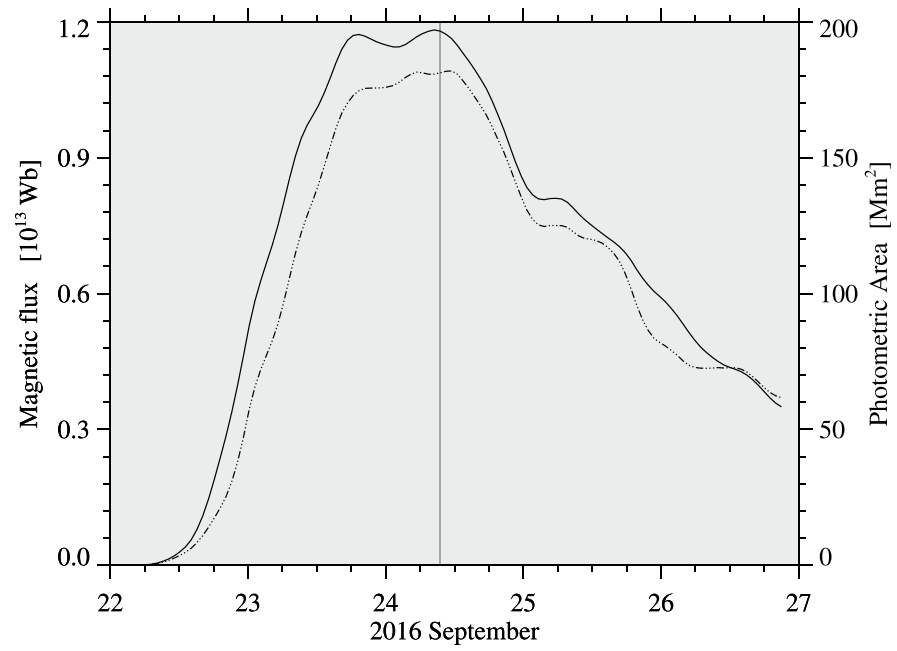

Fig. 6. Temporal evolution of the area (dash-dotted) and magnetic flux (solid) of the leading spot $\mathrm{P}_{1}$ using HMI continuum images and LOS magnetograms. The vertical line indicates the start of GREGOR observations.

field strength are taken at face value. We used linear regression to compute the growth and decay rates, because neither magnetic flux nor photometric area exhibited any indication for a parabolic growth or decay law. As seen in Fig. 6, the sunspot grew faster than it decayed. GREGOR observations (gray line) marked the start of the decay process. The flux decay rate of $0.33 \times 10^{13} \mathrm{~Wb}$ day $^{-1}$ was almost two and a half times lower than the growth rate of $0.80 \times 10^{13} \mathrm{~Wb}$ day $^{-1}$. The growth and decay rates for the leading spot $P_{1}$ agree with earlier results (e.g., Martínez Pillet 2002; Otsuji et al. 2011; Kubo et al. 2008). Similarly, the photometric area decay rate of $50 \mathrm{Mm}^{2}$ day $^{-1}$ was also about two and a half times lower than the growth rate of $110 \mathrm{Mm}^{2}$ day $^{-1}$.

\subsection{Horizontal proper motions}

The high-resolution GFPI image sequences allowed us to follow the horizontal proper motions around the sunspot $P_{1}$. We applied LCT (Sect. 2.2) to the broad-band image sequences, which covered a 40-min time period. The average flow map is depicted as rainbow-colored vectors in the right panel of Fig. 7. The expected global granular flow pattern with mesogranular cells was clearly visible within the quieter part of the FOV. One of the prominent flow features was a strong outward flow, that is, the moat flow partly encircling $P_{1}$. In addition, the typical divergence line (e.g., Deng et al. 2007) demarcated inflows in the inner and outflows in the outer penumbra. However, the moat flow was absent in the PS containing the elongated umbral core. Furthermore, the overall proper motions were diminished in that region. Only some distance away from PS, outward motions seem to continue but with much lower flow speeds. The moat flow appears to terminate at the nearest supergranular boundary, which was already reported in earlier studies (e.g., Verma et al. 2012).

The mean LCT velocity value over the whole FOV was $\bar{v}=0.42 \pm 0.26 \mathrm{~km} \mathrm{~s}^{-1}$ with the maximum velocity reaching $v_{\max }=1.57 \mathrm{~km} \mathrm{~s}^{-1}$. Furthermore, the 10th percentile velocity was $v_{10}=0.79 \mathrm{~km} \mathrm{~s}^{-1}$, and the median velocity was $v_{\text {med }}=$ $0.38 \mathrm{~km} \mathrm{~s}^{-1}$. Comparing these values with a previous study of Verma \& Denker (2011), where LCT was applied to Hinode $G$-band images containing a sunspot, the current velocities are somewhat lower, which is likely caused by the shorter cadence $\Delta t$ in the present study. The mean velocity in moat was $\bar{v}=0.49 \pm 0.27 \mathrm{~km} \mathrm{~s}^{-1}$, which is in agreement with values presented in previous studies (e.g., Sobotka \& Roudier 2007).

\subsection{Line-of-sight velocities}

Beyond the horizontal flow fields discussed in Sect. 3.2, GFPI spectra in the Fe I $\lambda 617.3 \mathrm{~nm}$ spectral line provided the photospheric LOS velocity in and around the leading sunspot (Sect. 2.2). The 40-min averaged velocity map depicted in the left panel of Fig. 7 was scaled between $\pm 1.2 \mathrm{~km} \mathrm{~s}^{-1}$. As expected, the Evershed flow appears around the sunspot penumbra as redand blueshifts, predominantly in the lower and upper parts of the spot. The Evershed flow starting in the penumbra continued as strong moat flow in the LCT map. The moat flow is also seen in the LOS velocity map, prominently at the limb-side penumbra. In that region we also noticed Evershed clouds (Cabrera Solana et al. 2006) in the time-lapse movie of LOS velocity maps. However, in the PS that faces the continuous flux emergence, the flows deviated from the usual penumbral flow pattern. The gap, which was created by the vanished penumbral filaments, displayed a flow pattern resembling that of granulation. The elongated umbral core in the PS possessed a velocity pattern similar to that of the umbra of sunspot $P_{1}$. However, the tip of the elongated umbral core exhibited a strong redshift, while the LCT velocity remained relatively low.

The GRIS spectra offered the possibility to infer both photospheric and chromospheric LOS velocities. Figure 8 depicts line-core intensity and LOS velocity maps for the three lines observed with GRIS. In general, these velocities are higher than those of the GFPI spectra. The maps for the Si I and Ca I lines, which represent the upper and the lower photosphere, respectively, show the granulation pattern as well as signatures of the Evershed flow. The inverse Evershed flow is visible in the He I map. A faint positive polarity patch, which is seen in the HMI magnetograms above $P_{1}$, appeared as a bright spot at coordinates $\left(93^{\prime \prime},-321^{\prime \prime}\right)$ in the Ca I line-core images and exhibited strong blueshifts. Velocities for the chromospheric He I line were higher than photospheric velocities. As expected the chromospheric He I map displayed a more filamentary structure. However, the He I line-core intensity did not show any (super)penumbral filaments above PS, showing a very perturbed penumbral structure. Bright features in the Ca I line-core intensity near PS exhibited blueshifts. However, the decaying sunspot $\mathrm{P}_{1}$ in the Si I LOS velocity map had very low velocities. In addition, strong downflows are present in the He I map, where the PS faces the EAR, but there is also significant asymmetry in coverage related to the inverse Evershed flow. In the Ca I LOS velocity map, the Lorentzian line-core fits failed in some parts of the umbra of $\mathrm{P}_{1}$, because the corresponding spectral line profiles were very shallow and significantly broadened by the strong magnetic field in the umbra.

\subsection{Line-of-sight magnetic field}

Magnetograms obtained with HMI give an overall impression of the magnetic field in and around the whole active region. To scrutinize the detailed magnetic structure of the leading spot, we used GRIS scans. We inverted the observed full-Stokes spectra with the SIR code (Sect. 2.2). The inversions were carried out for both the photospheric Si I and Ca I lines and for both scans, giving us an opportunity to study the temporal evolution and the height dependence of the magnetic field. The physical parameters obtained from the $\mathrm{Si}$ I and $\mathrm{Ca}$ I inversions are displayed in Figs. 9 and 10, respectively. Since the results for both scans differed only in detail, only the inferred parameters from the first 

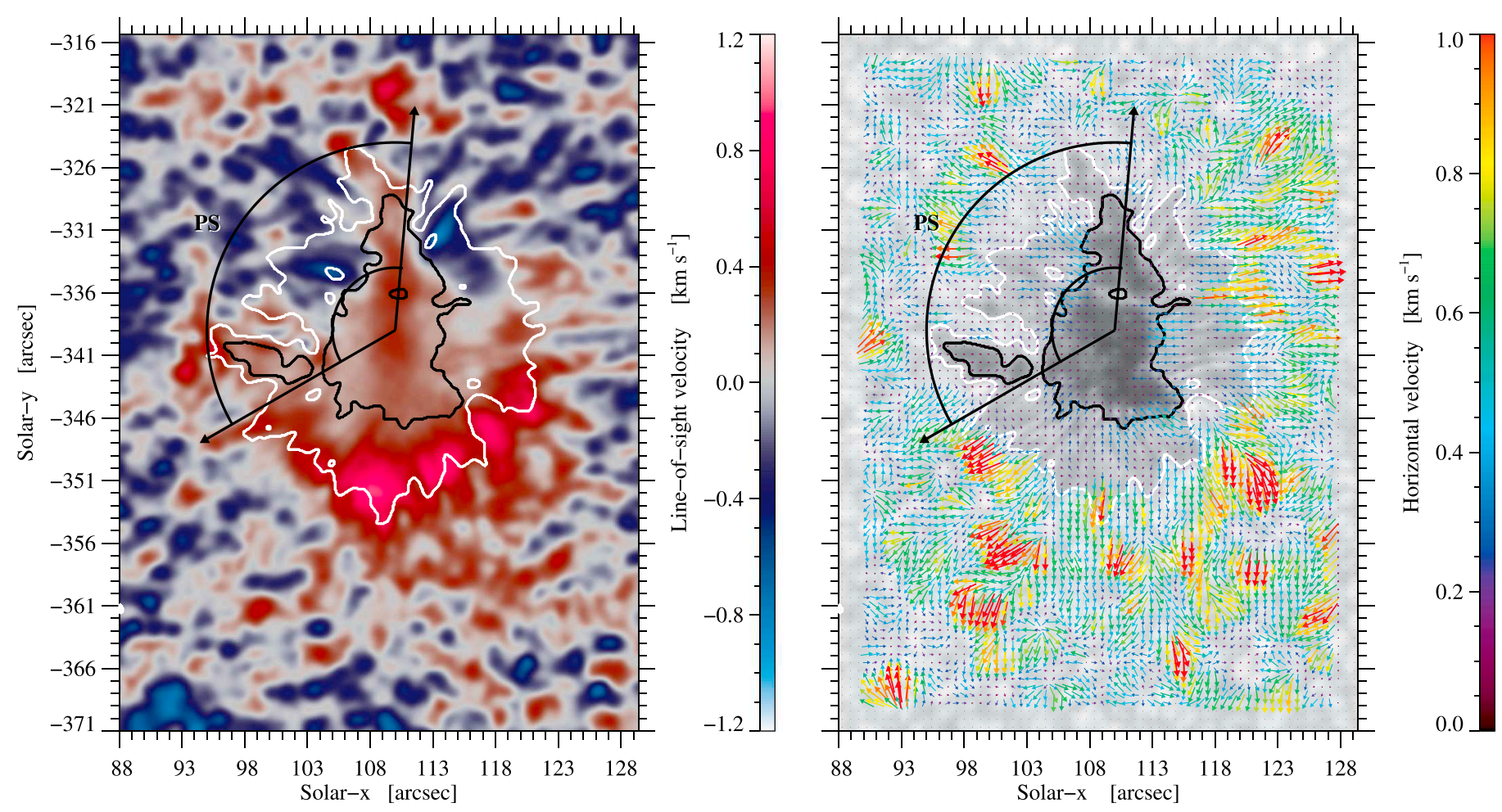

Fig. 7. Maps of GFPI averaged LOS velocity (left) and horizontal proper motions (right) around the leading spot $\mathrm{P}_{1}$, where color-coded vectors indicate magnitude and direction of the horizontal flows. The black contours mark the umbra-penumbra boundary, whereas the white contours denote the penumbra-granulation boundary. The contours shown here and in all subsequent figures are extracted from the GFPI broad-band image. The two black arcs mark the penumbral sector PS.

scan are shown for both lines. However, for both scans, the mean values of $B_{\mathrm{z}}, B_{\mathrm{hor}}$, and inclination $\gamma$ for umbra, elongated umbra in PS, PS without elongated umbra, and the remainder of the penumbra were compiled in Table 1.

The vertical magnetic flux density, $B_{\mathrm{z}}$, was positive for the leading spot, $\mathrm{P}_{1}$, as seen in the $\mathrm{Si}$ I line (Fig. 9). Small negativepolarity patches were found in the surroundings of $P_{1}$ in both lines. On smaller spatial scales, MMFs were present in the vicinity of the spot. They were mainly of type II (unipolar with the same polarity as the spot) with a few type III (unipolar with opposite polarity to the spot) and type I MMFs (bipolar with the inner foot-point of opposite polarity to the spot) interspersed. As noted in Table 1, the vertical component of the magnetic field for the elongated umbral core in the PS was higher than the rest of the penumbra and the PS, whereas the horizontal component was lowest for the elongated umbral core. The values of $B_{\text {hor }}$ for the PS facing the EAR was lower than the rest of the penumbra, but it contained some small-scale patches with high values. Even though barely noticeable in the continuum images, $B_{\text {hor }}$ had a significant magnetic flux density, which extended towards the EAR. The field inclination for the elongated umbral core in the PS was very similar to that of the umbra, that is, almost vertical (inclination of about $26^{\circ}$ ), and its structure can be easily traced in the map. For the rest of the penumbra, the horizontal flux density was high $B_{\text {hor }}=0.75 \pm 0.22 \mathrm{kG}$, the Evershed flow was strong, and the field inclination was around $50-70^{\circ}$ with the typical appearance of an "uncombed" penumbra.

The physical maps from the first scan of the Ca I line were compiled in Fig. 10. Since the Ca I line originates lower in the photosphere than the $\mathrm{Si}$ I line, $B_{\mathrm{Z}}$ had a smaller areal extent with only a few signs of MMFs. The MMFs are smaller in the $B_{z}$ map. The vertical and horizontal magnetic field components had higher values in Ca I line than Si I line. The elongated umbral core in the PS possessed a strong $B_{\mathrm{Z}}$ in the $\mathrm{Ca}$ I line as well. The lowest values are just above the gap intruding into the PS, which also had low $B_{\text {hor }}$ values. However, the upper part of the PS enclosed a patch with higher $B_{\text {hor values, but still lower than rest }}$ of the penumbra. The field inclination of the elongated umbral core in the PS was around $26^{\circ}$ in the Ca I line, with a distinct boundary setting it apart from the rest of the penumbra. The expansion of $B_{\text {hor }}$ was smaller compared to the Si I map. Similar to the Si I line, the Ca I map also contained very high $B_{\text {hor }}$ values for the rest of the penumbra. The field inclination for $P_{1}$ had a similar structure as in the Si I map with a mean of around $20^{\circ}$ for the umbra.

The inversion results and the magnetic properties of the second scan (not shown) were virtually identical to those of the first scan for both spectral lines, although the maps for the second scan encompassed a smaller FOV, and the seeing was not as good. In the continuum images for both lines, clockwise rotation of $P_{1}$ was apparent. The three types of MMFs were still present around the leading spot $P_{1}$. However, they became smaller as compared to the previous scan. The magnetic structures in $B_{\mathrm{z}}$ and $B_{\text {hor }}$ evolved little over the two hours. In the Si I maps, the elongated umbral core retained low values of $B_{\text {hor }}(=0.48 \pm 0.14 \mathrm{kG})$ but with increased values of $B_{\mathrm{z}}(=1.02 \pm 0.96 \mathrm{kG})$. A similar change in the magnetic field properties was also observed in the Ca I maps for the elongated umbral core with mean values $B_{\text {hor }}=0.75 \pm 0.22 \mathrm{kG}$ and $B_{\mathrm{z}}=1.43 \pm 0.20 \mathrm{kG}$.

In addition, the LOS velocities derived with the SIR code covered the range $\pm 3 \mathrm{~km} \mathrm{~s}^{-1}$. The velocity maps of the Si I line showed some indications of the Evershed effect, which was clearly seen in the Ca I line. The structures in the LOS velocity maps were very similar to the ones computed by simple 

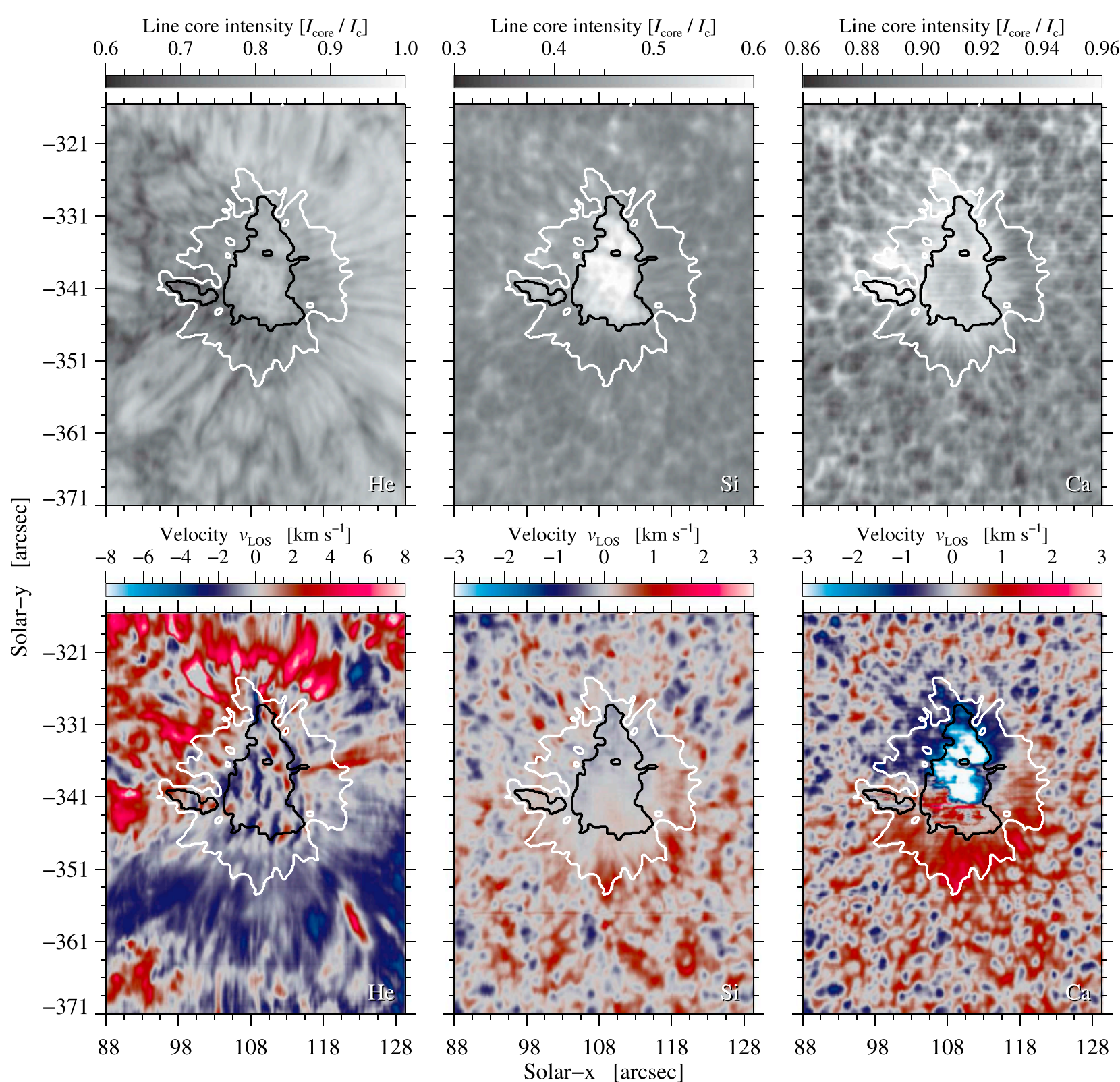

Fig. 8. Slit-reconstructed maps of the line-core intensity (top) and the LOS velocity (bottom) for the spectral lines He I (left), Si I (middle), and Ca I (right) observed with GRIS at 09:02 UT on 2016 September 24. The contours are the same as shown in Fig. 7. Simple line-fitting did not deliver proper fits for the Ca I line profiles in the umbra, which appear as conspicuous white patches in the respective velocity map.

line-core fitting (Fig. 8). However, the LOS velocities derived with the SIR code for the Ca I line slightly differed from the ones derived with line fitting. The inversion provided reasonable fits for the shallow and broad line profiles of the umbra. The FOV of the GRIS observations contains only the leading spot $P_{1}$, and it was possible to see the superpenumbra around it in the He I line core. As expected, the observed $Q^{-}, U_{-}$, and $V$-profiles show the extended magnetic field in the chromosphere (not shown here), but the small FOV was insufficient for us to comment on how the overlying chromospheric canopy affected the decaying penumbra.

The elongated umbral core in the PS has properties similar to the sunspot umbra. The average Stokes- $I$ and $V$-profiles in both the $\mathrm{Si}$ I and $\mathrm{Ca}$ I lines for the elongated umbral core and the $P_{1}$ umbra are displayed in Fig. 11. The intensity of the elongated umbral core exceeded that of the umbra. However, Stokes- $V$ exhibited a similar degree of polarization in both lines. The mean
$\mathrm{B}_{z}$ in Si I line for the elongated umbral core was $0.99 \pm 0.12 \mathrm{kG}$ and was lower in comparison to that of the umbra, $1.62 \pm 0.23 \mathrm{kG}$ . These values were higher in the $\mathrm{Ca}$ I line for both features, with $1.30 \pm 0.27 \mathrm{kG}$ for the elongated umbral core and $1.94 \pm 0.22 \mathrm{kG}$ for the umbra. The average inclination for the elongated umbral core was $26.2 \pm 8.8^{\circ}$ and $27.8 \pm 13.6^{\circ}$ in the $\mathrm{Si} \mathrm{I}$ and $\mathrm{Ca}$ I lines, respectively. For the umbra, the mean inclination was $21.2 \pm 8.3^{\circ}$ and $20.2 \pm 7.9^{\circ}$ in the $\mathrm{Si}$ I and $\mathrm{Ca}$ I lines, respectively. The values for $\mathrm{B}_{z}$ in the elongated umbral core increased from the first to the second scan for both spectral lines. However, as mentioned, the seeing was not good during the second scan, which could lead to a higher contribution from stray light. Additionally, the used inversion scheme does not account for the changing seeing conditions. Hence, the direct comparison of absolute values of the magnetic field is difficult. However, in comparison, the elongated umbral core has properties resembling those of the umbra in both scans, but they are not entirely identical. The values of $\mathrm{B}_{z}$ 

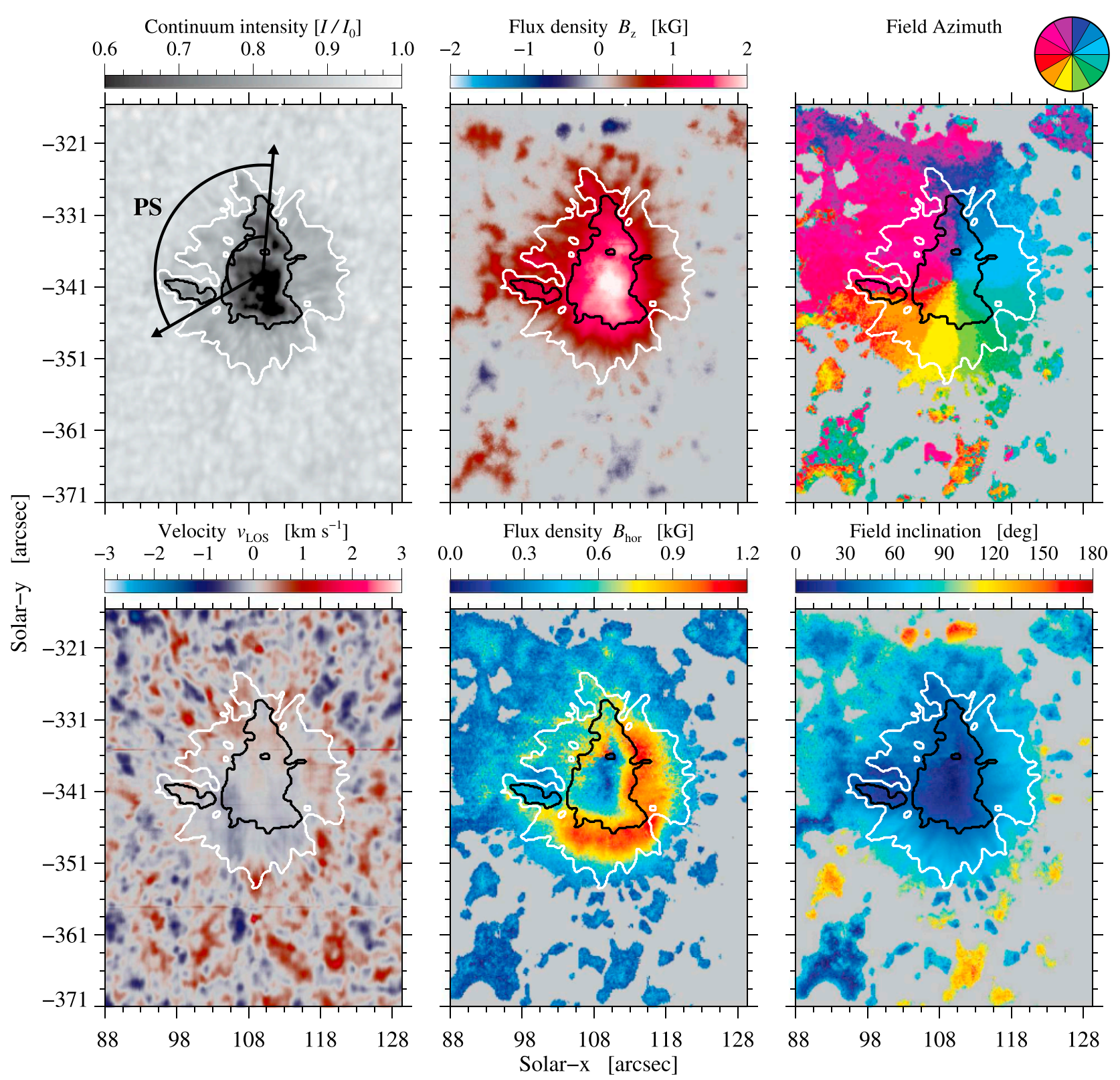

Fig. 9. Maps of physical parameters derived for the Si i line using the SIR code for the GRIS scan starting at 09:02 UT on 2016 September 24: normalized intensity $I / I_{0}$, vertical component of magnetic flux density $B_{\mathrm{z}}$, magnetic field azimuth $\phi$, Doppler velocity $v_{\mathrm{LOS}}$, horizontal component of magnetic flux density $B_{\text {hor }}$, and magnetic field inclination $\gamma$ (top-left to bottom-right). The polarization signal in the gray region is below the noise level. The two black arcs in the normalized intensity map mark the penumbral sector PS. We note that $B_{z}, \phi$, and $\gamma$ are in the local reference frame.

in $\mathrm{Ca}$ I for the elongated umbral core do reach the values as seen by Jurčák et al. (2015) during the penumbra formation. However, we do not have simultaneous Hinode data to confirm whether it fulfils the criteria proposed by Jurčák (2011) to establish a stable umbra-penumbra boundary.

To study the height dependence of the magnetic field, we divided the difference between the total $B$ and the vertical component $B_{\mathrm{Z}}$ of the magnetic flux density for the Si I and Ca I lines by the height difference of both lines for the first scan. The height for both lines was computed according to the method described in Balthasar \& Gömöry (2008) using the temperature map for both lines at $\tau=1$ obtained via SIR and contribution functions from two model atmospheres, that is, an umbral model (M4, Kollatschny et al. 1980) and a quiet-Sun model (t93-27, Schleicher 1976). The Si I line originates roughly about $350 \mathrm{~km}$ higher in the atmosphere than the Ca I line. The computed difference maps are compiled in Fig. 12.

The mean value of the $B$ gradients in the $P_{1}$ umbra of $-1.06 \pm 0.35 \mathrm{G} \mathrm{km}^{-1}$ and in the elongated umbral core of $-1.31 \pm$ $0.41 \mathrm{G} \mathrm{km}^{-1}$ was similar. The negative values indicate that the field strength decreases with height. The mean value for the penumbra of $-0.69 \pm 0.33 \mathrm{G} \mathrm{km}^{-1}$ was lower compared to both umbra and elongated umbral core. At the penumbra-granulation border, the field strength increased with height. In the gradient map for $B_{z}$, the trend for umbra and elongated umbral core was similar to the map for $B$. The mean value for the $B_{\mathrm{z}}$ gradient was negative in both the umbra of $-0.96 \pm 0.42 \mathrm{G} \mathrm{km}^{-1}$ and the elongated umbral core $-1.12 \pm 0.50 \mathrm{G} \mathrm{km}^{-1}$, which even slightly exceeded the umbral gradient. However, the gradient for the penumbra had small negative values with a mean of 

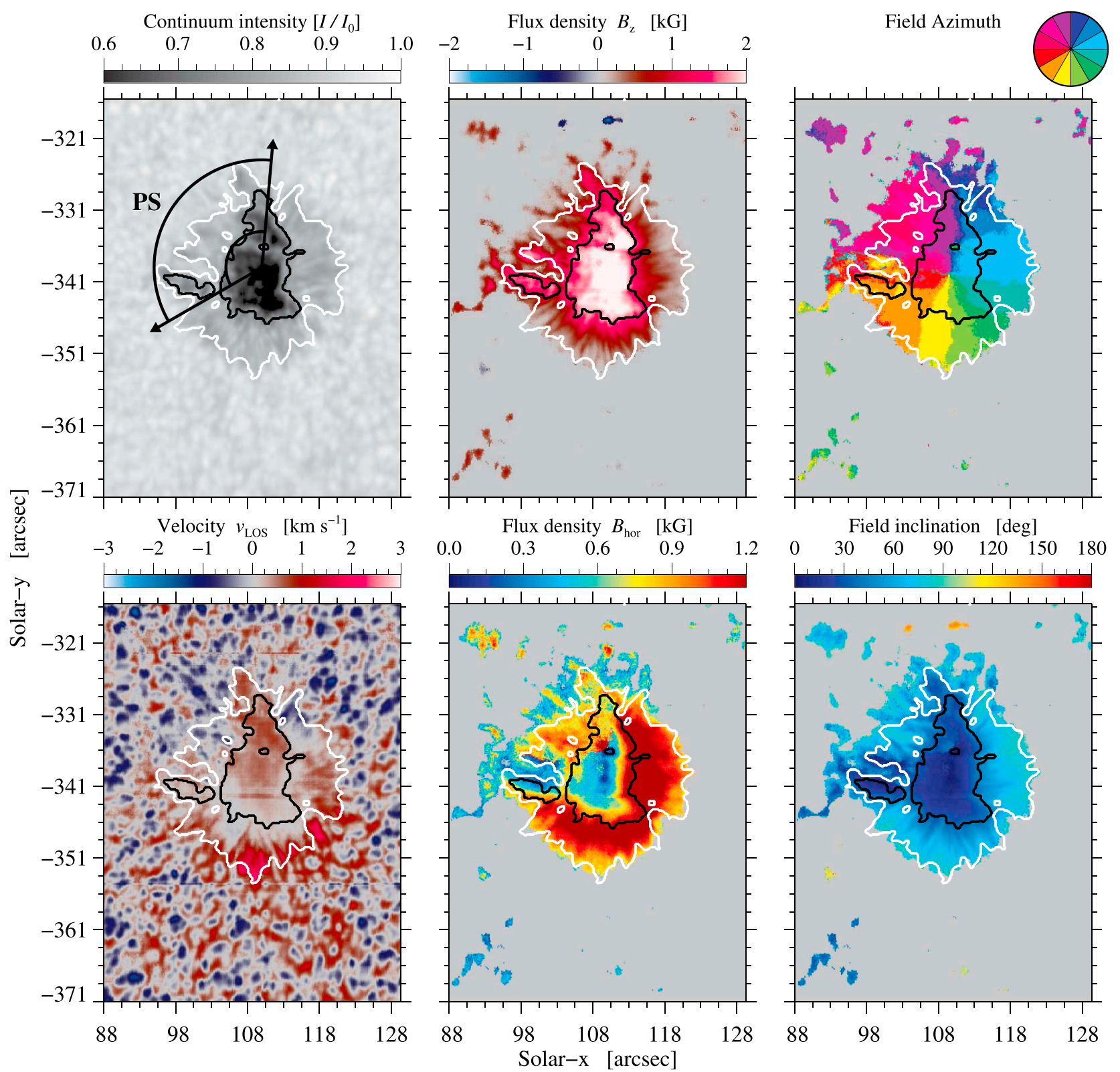

Fig. 10. Same as Fig. 9 but parameters derived for the Ca I line using the SIR code for the GRIS scan starting at 09:02 UT on 2016 September 24.

$-0.09 \pm 0.42 \mathrm{G} \mathrm{km}^{-1}$. The penumbral sector PS had a few patches with negative gradients. However, it also contained a positive gradient patch at the penumbra-granulation boundary. The magnetic field is less inclined higher in the atmosphere. Hence, the vertical component increases with the height leading to positive gradient values. In both gradient maps, the umbra and elongated umbral core exhibited a similar trend.

\section{Discussion and conclusions}

How and when does a sunspot decay? Does the penumbra simply disappear and do changes occur in magnetic field properties spontaneously? When the GREGOR observations started, the leading spot began to decay, which became evident, when two light bridges appeared in the umbra. Light bridges are an indication of impending sunspot fragmentation (Vazquez 1973). One of the light bridges disappeared and an elongated, dark umbral core formed at its edge within the PS. The penumbral filaments in this sector did not simply vanish but formed dark features with umbra-like velocity and magnetic field properties. Light bridges are suggested as a trigger for penumbra formation by Rezaei et al. (2012). However, in our case the light-bridge initiated the transformation of penumbral filaments into an elongated, dark umbral core. This conversion ultimately led to the disappearance of the penumbral filaments at this location.

With the GREGOR telescope and its instruments, we have access to the three-dimensional flow field in and around the leading sunspot. The usual outward directed moat flow was present around the spot in horizontal flow maps. However, it was absent within the PS with the elongated, dark umbral core. Here, the LCT velocities were low indicating constrained horizontal plasma motions. The photospheric GFPI and GRIS LOS velocity maps reveal the Evershed flow around the sunspot. However, this distinct flow pattern was absent in the above mentioned PS. Signatures of convective motions were noticeable in the LOS velocity maps in the region between the elongated, dark umbral core and the neighboring penumbral filaments. The umbral core 
Table 1. Average magnetic field properties derived from SIR for umbra, elongated umbral core, penumbral sector PS, and the remainder of the penumbra for both the Si I and the Ca I spectral lines and for both GRIS scans.

\begin{tabular}{|c|c|c|c|}
\hline Features & $\begin{array}{c}B_{\mathrm{z}} \pm \sigma_{B_{\mathrm{z}}} \\
{[\mathrm{kG}]}\end{array}$ & $\begin{array}{c}B_{\text {hor }} \pm \sigma_{B_{\text {hor }}} \\
{[\mathrm{kG}]}\end{array}$ & $\begin{array}{c}\gamma \pm \sigma_{\gamma} \\
{[\operatorname{deg}]}\end{array}$ \\
\hline $\begin{array}{l}\text { Umbra } \\
\text { Elongated Umbra } \\
\text { Penumbral Sector } \\
\text { Penumbra }\end{array}$ & $\begin{array}{l}1.62 \pm 0.23 \\
0.99 \pm 0.12 \\
0.83 \pm 0.24 \\
0.59 \pm 0.42 \\
\end{array}$ & $\begin{array}{c}\text { Si I }- \text { Scan I } \\
0.61 \pm 0.22 \\
0.48 \pm 0.15 \\
0.57 \pm 0.15 \\
0.75 \pm 0.22 \\
\end{array}$ & $\begin{array}{r}21.25 \pm 8.38 \\
26.15 \pm 8.85 \\
35.31 \pm 10.30 \\
56.80 \pm 14.53 \\
\end{array}$ \\
\hline $\begin{array}{l}\text { Umbra } \\
\text { Elongated Umbra } \\
\text { Penumbral Sector } \\
\text { Penumbra }\end{array}$ & $\begin{array}{l}1.53 \pm 0.20 \\
1.02 \pm 0.10 \\
0.76 \pm 0.24 \\
0.61 \pm 0.41\end{array}$ & $\begin{array}{c}\text { Si I }- \text { Scan II } \\
0.65 \pm 0.22 \\
0.48 \pm 0.13 \\
0.55 \pm 0.16 \\
0.70 \pm 0.21\end{array}$ & $\begin{array}{l}23.29 \pm 8.60 \\
24.99 \pm 6.62 \\
37.04 \pm 11.50 \\
54.50 \pm 14.96\end{array}$ \\
\hline $\begin{array}{l}\text { Umbra } \\
\text { Elongated Umbra } \\
\text { Penumbral Sector } \\
\text { Penumbra }\end{array}$ & $\begin{array}{l}1.94 \pm 0.22 \\
1.30 \pm 0.27 \\
0.94 \pm 0.43 \\
0.61 \pm 0.50\end{array}$ & $\begin{array}{c}\mathrm{Ca} I-\text { Scan I } \\
0.71 \pm 0.27 \\
0.69 \pm 0.27 \\
0.74 \pm 0.22 \\
1.05 \pm 0.24\end{array}$ & $\begin{array}{c}20.20 \pm 7.86 \\
27.80 \pm 13.65 \\
40.81 \pm 17.62 \\
64.57 \pm 16.38\end{array}$ \\
\hline $\begin{array}{l}\text { Umbra } \\
\text { Elongated Umbra } \\
\text { Penumbral Sector } \\
\text { Penumbra }\end{array}$ & $\begin{array}{l}1.87 \pm 0.22 \\
1.43 \pm 0.20 \\
0.80 \pm 0.40 \\
0.63 \pm 0.51\end{array}$ & $\begin{array}{c}\mathrm{Ca} \text { I }- \text { Scan II } \\
0.78 \pm 0.29 \\
0.75 \pm 0.22 \\
0.83 \pm 0.22 \\
1.02 \pm 0.24\end{array}$ & $\begin{array}{c}22.77 \pm 9.17 \\
27.68 \pm 9.89 \\
48.62 \pm 17.41 \\
63.16 \pm 18.31\end{array}$ \\
\hline
\end{tabular}

displayed low LOS velocities with an average velocity near zero. This indicates that the elongated, dark umbral core appearing in place of penumbral filaments had flow properties similar to an umbra in both horizontal and LOS velocities maps.

The magnetic field properties of the decaying PS differed from the usual penumbra. The elongated, dark umbral core possessed a low horizontal field component, but a strong vertical magnetic field component. Furthermore, the magnetic field inclination was also close to zero. This indicated that the field lines in this location of penumbra were nearly vertical and that the extruding umbral core resembled a typical sunspot umbra. The magnetic field properties extracted from the Si I and $\mathrm{Ca}$ I lines were slightly different but followed a similar trend. The vertical and horizontal magnetic field components in the lower photosphere ( $\mathrm{Ca}$ I line) were more confined and stronger. In the spectropolarimetic observations of a decaying sunspot Bellot Rubio et al. (2008) found small-scale inhomogeneities in the area surrounding a naked umbra. These finger-like features had weak and nearly horizontal magnetic field. They suggested that the penumbra at photospheric heights will disappear, when the penumbral field lines, which no longer carry strong Evershed flows, rise to the chromosphere. In our data, even though not all penumbral filaments vanished, we noticed that the PS facing the flux emergence site did not display the usual Evershed and moat flow. In addition, the horizontal magnetic field was weaker for the entire PS. Although, the rest of the sunspot penumbra showed the typical flow and magnetic field properties, including the presence of small-scale moving magnetic features.

Rapid penumbral decay is closely related to flares (Wang et al. 2004; Deng et al. 2005) and much faster (a few minutes) than the usual decay process. Beauregard et al. (2012) observed penumbral decay and umbral strengthening after an
X-class solar flare. For the same solar flare, Wang et al. (2012b) investigated the response of the photospheric magnetic field. They found evidence of a rapid and irreversible enhancement of the horizontal magnetic field at the flaring magnetic polarity inversion line. They related the strengthening of horizontal magnetic field to newly formed low-lying fields resulting from tether-cutting reconnection (Moore et al. 2001). Rapid rearrangement of penumbral magnetic field lines in flaring $\delta$-sunspots was the focus of the work by Wang et al. (2012a). They suggested that the magnetic field became more vertical due to restructuring associated with flares and concluded that this led to a transformation of dark penumbral filaments to faculae. In the present study, we find a similar restructuring of the horizontal component of the magnetic field and a strengthening of the vertical magnetic field component as well as the appearance of an elongated, dark umbral core in PS. However, the duration of the transformation took place on much longer time-scales than those inferred by Wang et al. (2012a). Thus, also slow changes of the magnetic field topology within an active region, for example, introduced by flux emergence, can induce the transformation of penumbral to umbral magnetic fields.

How does the surrounding magnetic field affect sunspot structure and evolution? Looking just beyond the FOV covered by GREGOR observations, we noticed that the decaying penumbral sector faced a region of continuous flux emergence (EAR). Recently, Murabito et al. (2017) observed the formation of stable penumbra at the site facing the flux emergence. Their observations agree with the scenario proposed by Lim et al. (2013), which suggests the pre-existing chromospheric canopy field assists in the formation of the penumbral filaments in the flux emergence region. However, in our case the penumbral sector facing the flux emergence site decayed. The side of the sunspot facing the flux emergence site was stretched out towards the EAR. In addition, the approaching polarity of the EAR was the same as that of the decaying sunspot. The same polarity flux might have influenced the magnetic canopy of the decaying sunspot disrupting the stable horizontal penumbral field (Künzel 1969).

In time-lapse movies of HMI continuum images, HMI magnetograms, and AIA $\lambda 171 \mathrm{~nm}$ images, we observed that the leading polarity of the EAR continuously pushed the elongated, dark umbral core and the whole PS of the leading spot $P_{1}$, resulting in rotation of the entire sunspot. In the sketch represented in Fig. 5, we summarized these dynamical properties seen in the region. Botha et al. (2011) studied the decay process of large magnetic flux tubes, such as sunspots, on a supergranular scale. Their nonlinear cylindrical simulation consisted of a well-defined central flux tube and an annular convection cell surrounding it. They found that the evolving azimuthal nonlinear convection in the annular cells breaks the flux tube, and the magnetic flux from the central flux tube then slips between these fragmented cells. This leads to a reduction of magnetic pressure and an increase of convection in the central flux tube. The convection inside the tube grows until the spot starts to disintegrate. Hence, they concluded that the decay of the central flux tube depends on the convection in the surroundings, which can add or remove flux from the central tube. In addition, convection in the vicinity of the spot can change the shape of the central flux tube. Our decaying sunspot was in the vicinity of a newly emerging flux system, where the separating polarities of the EAR pushed and rotated the DAR. Our observations imply that the decay of penumbra involved the interaction of two flux systems, which changed the surrounding motions. We conclude that the continuous flux emergence in the region likely altered the convection 

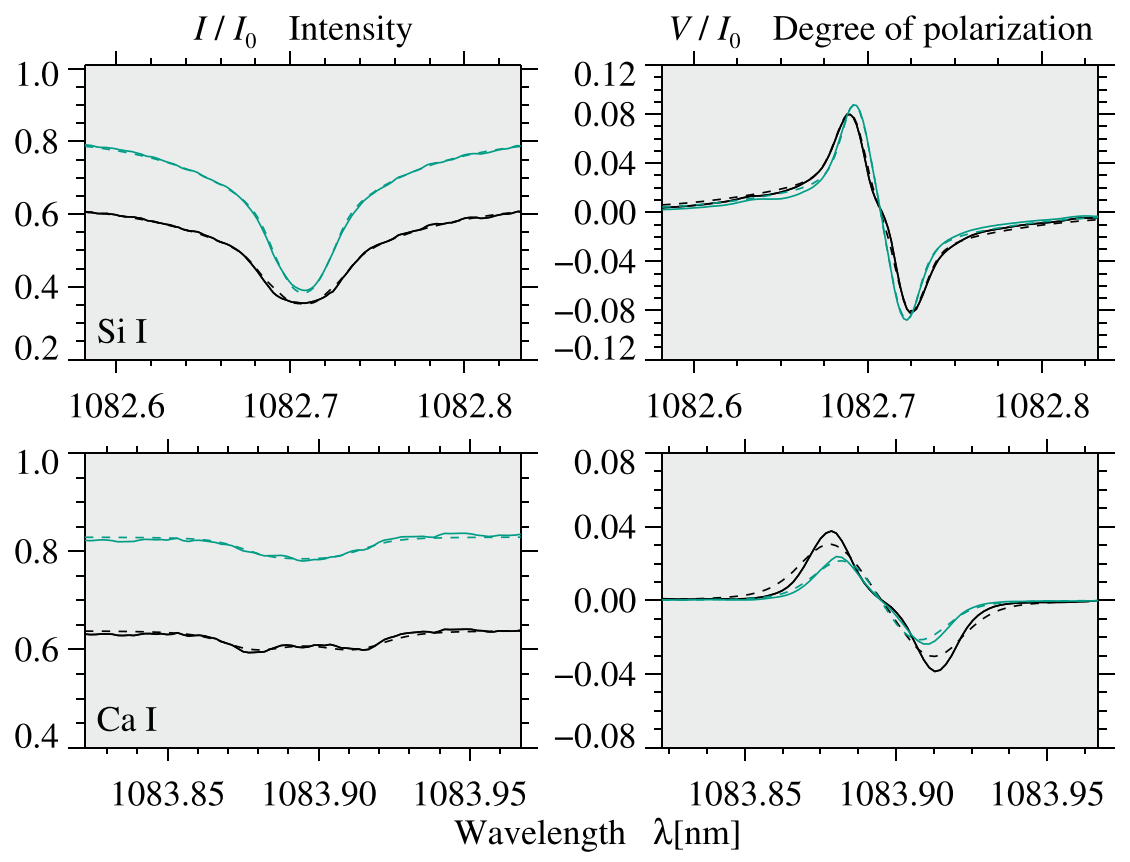

Fig. 11. Average Stokes- $I$ and $-V$ profiles (solid) for the elongated umbral core (green) and the umbra of $\mathrm{P}_{1}$ (black) for the Si I (top) and Ca I (bottom) lines. The SIR results for these profiles are shown in same colors but as dashed lines.

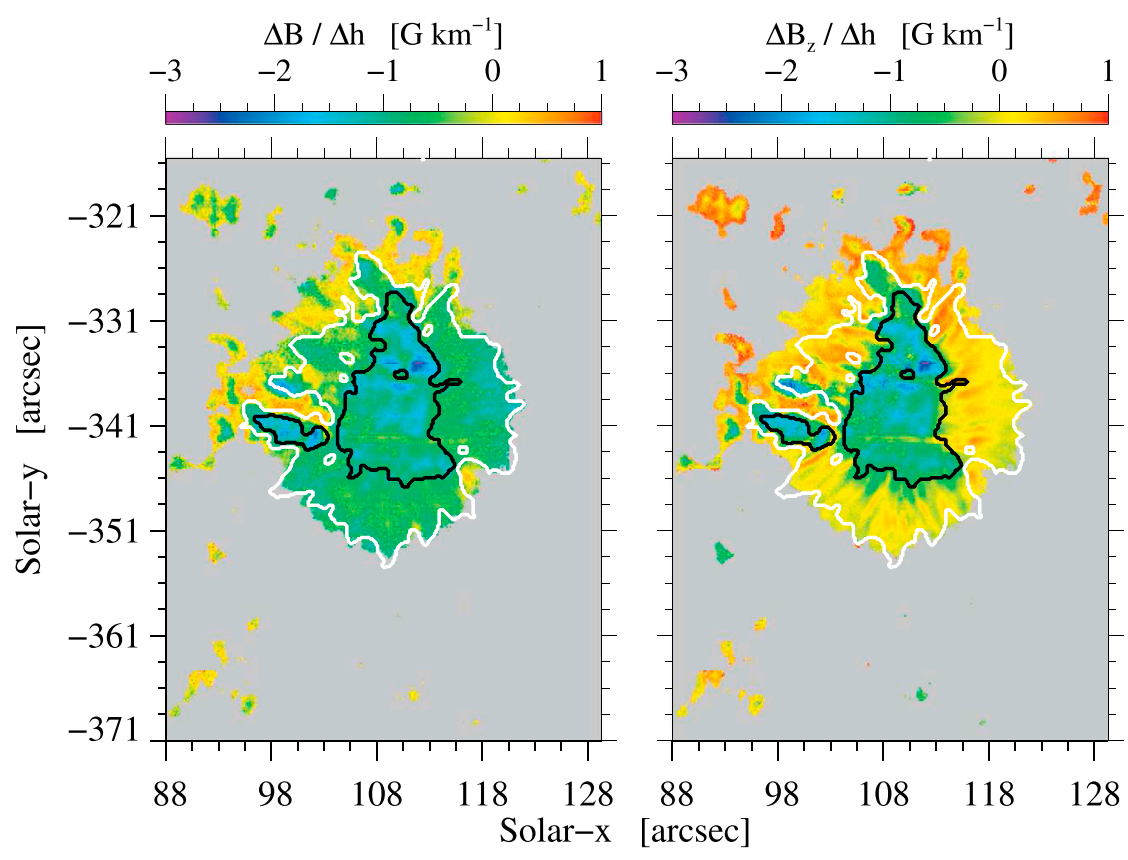

Fig. 12. Difference of the total magnetic field strength (left) and vertical component of the magnetic field (right) from the spectral lines Si I and Ca I, respectively, divided by the height difference. pattern in the sunspot's surrounding, resulting in an accelerated decay of penumbral filaments, and led to significant rotation of the leading sunspot in the DAR.

The relation between penumbra formation and the surrounding flux emergence remains contradictory. Schlichenmaier et al. (2010, 2012) and Rezaei et al. (2012) found that a sunspot penumbra only develops in a region with stable conditions. In their studies, the side of the sunspot facing the flux emergence site did not develop a penumbra. However, Lim et al. (2013) and Murabito et al. (2017) found penumbra formation on the side of the sunspot facing the flux emergence region. Both agreed that the overlying chromospheric canopy plays a role in the formation of a penumbra. In our sunspot, the penumbral sector facing the flux emergence site was already in the decay phase at the start of the GREGOR observations. In addition, we did not perform a detailed analysis of the chromospheric magnetic field because the FOV was not sufficiently large. Hence, it is difficult to pin-point if it initiated and/or assisted the decay process. However, we can state that the magnetic and flow changes caused by the ongoing flux emergence did affect the penumbral decay process around the sunspot and likely changed the stable or "quiet" conditions that are required to sustain a stable penumbra.

In the current work, we present high-resolution spectroscopic and polarimetric observations of a decaying sunspot. At the time of the GREGOR observations, the sunspot contained light bridges indicating the start of its fragmentation. After the decay of one of the light bridges, an elongated, dark umbral core appeared at its edge within the decaying penumbral sector. The flow and magnetic field properties of this penumbral sector exhibited weak Evershed flow, moat flow, and horizontal magnetic fields. Concurrently, a new flux system emerged 
in the already established flux system. The interaction between emerging and already established flux system played an important role in penumbral decay and additionally resulted in rotation of the leading sunspot. This hints at a subphotospheric interaction of both flux systems releasing twist and writhe in the DAR. In future work, we will analyze and follow the evolution of the active region based on the full set of high-resolution data acquired with GREGOR, VTT, NST, and DST, which was obtained over several days during the coordinated campaign. As of yet, we have only discussed the photospheric magnetic field properties of active region NOAA 12597. In a following step, we will include the inversion results of the chromospheric He I triplet. These high-resolution observations are needed to provide a complete and comprehensive picture of the sunspot decay process.

Acknowledgements. The 1.5-meter GREGOR solar telescope was built by a German consortium under the leadership of the Kiepenheuer-Institut für Sonnenphysik in Freiburg with the Leibniz-Institut für Astrophysik Potsdam, the Institut für Astrophysik Göttingen, and the Max-Planck-Institut für Sonnensystemforschung in Göttingen as partners, and with contributions from the Instituto de Astrofísica de Canarias and the Astronomical Institute of the Academy of Sciences of the Czech Republic. The National Solar Observatory is operated by the Association of Universities for Research in Astronomy under a cooperative agreement with the National Science Foundation. SDO HMI and AIA data are provided by the Joint Science Operations Center - Science Data Processing. MS is supported by the Czech Science Foundation under the grant 14-04338S. This study is supported by the European Commission's FP7 Capacities Programme under the Grant Agreement number 312495. R.R. acknowledges financial support from the Spanish Ministry of Economy and Competitiveness through project AYA2014-60476-P. SJGM is grateful for financial support from the Leibniz Graduate School for Quantitative Spectroscopy in Astrophysics, a joint project of AIP and the Institute of Physics and Astronomy of the University of Potsdam and he acknowledges support from the project VEGA 2/0004/16.

\section{References}

Balthasar, H. 2006, A\&A, 449, 1169

Balthasar, H., \& Gömöry, P. 2008, A\&A, 488, 1085

Balthasar, H., Beck, C., Gömöry, P., et al. 2013, Centr. Eur. Astrophys. Bull., 37, 435

Balthasar, H., Gömöry, P., González Manrique, S. J., et al. 2016, Astron. Nachr., 337,1050

Beauregard, L., Verma, M., \& Denker, C. 2012, Astron. Nachr., 333, 125

Bello González, N., \& Kneer, F. 2008, A\&A, 480, 265

Bellot Rubio, L. R., Tritschler, A., \& Martínez Pillet, V. 2008, ApJ, 676, 698

Botha, G. J. J., Rucklidge, A. M., \& Hurlburt, N. E. 2011, ApJ, 731, 108

Bumba, V. 1963, Bull. Astr. Inst. Czechosl., 14, 91

Cabrera Solana, D., Bellot Rubio, L. R., Beck, C., \& del Toro Iniesta, J. C. 2006 , ApJ, 649, L41

Collados, M. 1999, in Third Advances in Solar Physics Euroconference: Magnetic Fields and Oscillations, eds., B. Schmieder, A. Hofmann, \& J. Staude, ASP Conf. Ser., 184, 3

Collados, M., López, R., Páez, E., et al. 2012, Astron. Nachr., 333, 872

Deng, N., Liu, C., Yang, G., Wang, H., \& Denker, C. 2005, ApJ, 623, 1195

Deng, N., Choudhary, D. P., Tritschler, A., et al. 2007, ApJ, 671, 1013

Denker, C., Balthasar, H., Hofmann, A., Bello González, N., \& Volkmer, R. 2010, in Proc. SPIE, 7735, 77356M

Denker, C., von der Lühe, O., Feller, A., et al. 2012, Astron. Nachr., 333, 810

Denker, C., Kuckein, C., Verma, M., et al. 2018, ApJS, 236, 5

González Manrique, S. J., Kuckein, C., Pastor Yabar, A., et al. 2016, Astron. Nachr., 337, 1057

Hanisch, R. J., Farris, A., Greisen, E. W., et al. 2001, A\&A, 376, 359

Harvey, K., \& Harvey, J. 1973, Sol. Phys., 28, 61
Ichimoto, K., Lites, B., Elmore, D., et al. 2008, Sol. Phys., 249, 233

Jurčák, J. 2011, A\&A, 531, A118

Jurčák, J., Bello González, N., Schlichenmaier, R., \& Rezaei, R. 2015, A\&A, 580, L1

Jurčák, J., Bello González, N., Schlichenmaier, R., \& Rezaei, R. 2017, A\&A, 597, A60

Kollatschny, W., Wiehr, E., Stellmacher, G., \& Falipou, M. A. 1980, A\&A, 86, 245

Kosugi, T., Matsuzaki, K., Sakao, T., et al. 2007, Sol. Phys., 243, 3

Kubo, M., Lites, B. W., Shimizu, T., \& Ichimoto, K. 2008, ApJ, 686, 1447

Kuckein, C., Denker, C., Verma, M., et al. 2017, IAU Symp., 327, 20

Künzel, H. 1969, Astron. Nachr., 291, 265

Leka, K. D., \& Skumanich, A. 1998, ApJ, 507, 454

Lemen, J. R., Title, A. M., Akin, D. J., et al. 2012, Sol. Phys., 275, 17

Lim, E.-K., Yurchyshyn, V., Goode, P., \& Cho, K.-S. 2013, ApJ, 769, L18

Löfdahl, M. G. 2002, in Image Reconstruction from Incomplete Data, eds. P. J. Bones, M. A. Fiddy, \& R. P. Millane, Proc. SPIE, 4792, 146

Martínez Pillet, V. 2002, Astron. Nachr., 323, 342

McIntosh, P. S. 1981, in The Physics of Sunspots, eds. L. E. Cram, \& J. H. Thomas, 7

Meyer, F., Schmidt, H. U., Wilson, P. R., \& Weiss, N. O. 1974, MNRAS, 169, 35

Moore, R. L., Sterling, A. C., Hudson, H. S., \& Lemen, J. R. 2001, ApJ, 552, 833 Murabito, M., Romano, P., Guglielmino, S. L., \& Zuccarello, F. 2017, ApJ, 834, 76

Neckel, H. \& Labs, D. 1984, Sol. Phys., 90, 205

November, L. J., \& Simon, G. W. 1988, ApJ, 333, 427

Otsuji, K., Kitai, R., Ichimoto, K., \& Shibata, K. 2011, PASJ, 63, 1047

Pesnell, W. D., Thompson, B. J., \& Chamberlin, P. C. 2012, Sol. Phys., 275, 3

Petrovay, K., \& van Driel-Gesztelyi, L. 1997, Sol. Phys., 176, 249

Pierce, A. K., \& Slaughter, C. D. 1977, Sol. Phys., 51, 25

Puschmann, K. G., Denker, C., Kneer, F., et al. 2012, Astron. Nachr., 333, 880

Reiners, A., Mrotzek, N., Lemke, U., Hinrichs, J., \& Reinsch, K. 2016, A\&A 587, A65

Rempel, M. 2015, ApJ, 814, 125

Rezaei, R., Bello González, N., \& Schlichenmaier, R. 2012, A\&A, 537, A19

Rucklidge, A. M., Schmidt, H. U., \& Weiss, N. O. 1995, MNRAS, 273, 491

Ruiz Cobo, B., \& del Toro Iniesta, J. C. 1992, ApJ, 398, 375

Sainz Dalda, A., \& Martínez Pillet, V. 2005, ApJ, 632, 1176

Scherrer, P. H., Schou, J., Bush, R. I., et al. 2012, Sol. Phys., 275, 207

Schleicher, H. 1976, PhD thesis, University Göttingen, 1976

Schlichenmaier, R., Rezaei, R., Bello González, N., \& Waldmann, T. A. 2010, A\&A, 512, L1

Schlichenmaier, R., Rezaei, R., \& González, N. B. 2012, in 4th Hinode Science Meeting: Unsolved Problems and Recent Insights, eds. L. Bellot Rubio,

F. Reale, \& M. Carlsson (San Francisco: ASP), ASP Conf. Ser., 455, 61

Schmidt, W., Stix, M., \& Wöhl, H. 1999, A\&A, 346, 633

Schmidt, W., von der Lühe, O., Volkmer, R., et al. 2012, Astron. Nachr., 333, 796

Schou, J., Scherrer, P. H., Bush, R. I., et al. 2012, Sol. Phys., 275, 229

Sobotka, M., \& Roudier, T. 2007, A\&A, 472, 277

Solanki, S. K. 2003, A\&ARv, 11, 153

Tildesley, M. J., \& Weiss, N. O. 2004, MNRAS, 350, 657

Tsuneta, S., Ichimoto, K., Katsukawa, Y., et al. 2008, Sol. Phys., 249, 167

van Noort M., Rouppe van der Voort, L., \& Löfdahl, M. G. 2005, Sol. Phys., 228,

191

Vazquez, M. 1973, Sol. Phys., 31, 377

Verma, M., \& Denker, C. 2011, A\&A, 529, A153

Verma, M., Balthasar, H., Deng, N., et al. 2012, A\&A, 538, A109

Verma, M., Steffen, M., \& Denker, C. 2013, A\&A, 555, A136

Verma, M., Denker, C., Böhm, F., et al. 2016, Astron. Nachr., 337, 1090

von der Lühe, O. 1998, New Astron. Rev., 42, 493

Wang, H., Liu, C., Qiu, J., et al. 2004, ApJ, 601, L195

Wang, H., Deng, N., \& Liu, C. 2012a, ApJ, 748, 76

Wang, S., Liu, C., Liu, R., et al. 2012b, ApJ, 745, L17

Watanabe, H., Kitai, R., \& Otsuji, K. 2014, ApJ, 796, 77

Wells, D. C., Greisen, E. W., \& Harten, R. H. 1981, A\&AS, 44, 363

Wöger, F., \& von der Lühe, O. 2008, in Advanced Software and Control for Astronomy II, eds., A. Bridger, \& N. M. Radziwill, Proc. SPIE, 7019, 70191E Wöger, F., von der Lühe, O., \& Reardon, K. 2008, A\&A, 488, 375 